\title{
Novel Intercell Interference Mitigation Algorithms for Multicell OFDMA Systems with Limited Base Station Cooperation
}

\author{
Jia Shi, Member, IEEE, Lie-Liang Yang, Fellow, IEEE, and Qiang Ni, Senior Member, IEEE
}

\begin{abstract}
Resource allocation in multicell downlink orthogonal frequency division multiple-access (OFDMA) systems is investigated, where BSs first independently carry out subcarrierallocation and then mitigate intercell interference (InterCI) with the aid of very limited base station (BS) cooperation. Two novel InterCI mitigation algorithms are proposed. The first one is the distributed decision making assisted cooperation (DDMC) algorithm, and the second one is the centralized decision making assisted cooperation (CDMC) algorithm. When employing the DDMC algorithm, each BS independently makes the InterCI mitigation decisions. By contrast, when employing the CDMC algorithm, the centralized InterCI mitigation decisions are made with the aid of the cell-edge users' discrete InterCI information sharing among BSs. While both the algorithms motivate to maximize the spectral-efficiency (sum rate), the CDMC algorithm also aims to maximize the frequency reuse factor. In this paper, we study and compare the performance, including spectralefficiency of cell-edge users, frequency reuse factor, overhead, etc., of the multicell downlink OFDMA systems employing the proposed and other InterCI mitigation algorithms. Our studies show that both the DDMC and CDMC algorithms can achieve better spectral-efficiency performance than the existing on-off power (OOP) algorithm. Moreover, the CDMC algorithm is capable of achieving the performance close to the upper-bound attained by the so-called full InterCI information assisted decision making (FIIDM) algorithm, which uses exhaustive search to determine the InterCI mitigation decisions. Additionally, the CDMC algorithm is demonstrated to have the highest frequency reuse factor in addition to its spectral-efficiency advantage.
\end{abstract}

Index Terms-Resource allocation, subcarrier-allocation, multicell, OFDMA, intercell interference, base station cooperation, optimization.

\section{INTRODUCTION}

Orthogonal frequency division multiple access (OFDMA) has emerged as one of the key techniques for high-speed broadband wireless communications. In the literature, resource allocation in single-cell OFDMA systems have been widely investigated, especially, in association with subcarrierallocation [1-6]. However, mobile communication systems are typically multicell systems with frequency spectrum reused in geographic areas. Moreover, towards the future generations of wireless systems, unity of frequency reuse is desired. In this case, users may experience severe intercell interference

Copyright (c) 2015 IEEE. Personal use of this material is permitted. However, permission to use this material for any other purposes must be obtained from the IEEE by sending a request to pubs-permissions@ieee.org. J. Shi and Q. Ni are with the School of Computing and Communications, Lancaster University, LA1 4WA, UK; L.-L. Yang is with School of Electronics and Computer Science, University of Southampton, SO17 1BJ, UK. (E-mail: j.shi@lancaster.ac.uk, 1ly@ecs.soton.ac.uk, q.ni@lancaster.ac.uk.)
(InterCI), resulting in significant performance degradation, if it is not efficiently managed.

In multicell communications, resource allocation approaches proposed in the literature may be categorized into two classes, namely, centralized and distributed resource allocation, based on where and how the resource allocation is carried out. Specifically, in centralized resource allocation, central control units are used to collect the required information, which are also responsible for managing and allocating resources jointly to all users in all cells. Centralized resource allocation may consume the enormous resources, which could be exploited for data transmission, for information exchange and system controlling [7]. In the literature, there are a range of references, including [7-12], having proposed and studied the centralized resource allocation in multicell OFDMA systems. In [8], the authors have proposed a load matrix approach for jointly managing both the InterCI and the intracell interference (IntraCI) experienced by users. In [9], a NP-hard joint resource allocation problem for a twocell OFDMA system has been approximated by a weighted sum throughput maximization problem. Using the geometric programming approach to transform the original mixed integer nonconvex problems, the authors in $[7,11]$ have proposed the sub-optimal subcarrier- and power-allocation solutions in the downlink OFDMA networks with BS coordination. By contrast, in [12], the authors have dealt with the IntraCI of a subcarrier reused OFDMA networks.

In distributed resource allocation, every BS independently allocates its resources, usually, based only on the intracell channel information and the interference measured locally. In comparison with the centralized approaches, distributed resource allocation has the main advantages of fast response to dynamic resource environments, fast time-varying channels, and of low complexity for implementation. Distributed resource allocation in multicell OFDMA systems has been widely studied, as evidenced, e.g., by [13-18]. The distributed resource allocation scheme proposed in [13] has considered jointly subcarrier, bit and power allocation in multicell OFDMA systems. In [14], the authors have studied the distributed subcarrier- and power-allocation in the multicell OFDMA networks with cognitive radio functionality. In [15], a distributed power-allocation scheme has been proposed for the multicell multiple input single output (MISO) OFDMA networks, where the channel state information (CSI) of all users is shared among the BSs. Very recently, interference aware resource allocation has drawn the attention $[17,18]$. 
It can be understood that, in order to combat the InterCI existing in multicell OFDMA systems, one may employ sophisticated InterCI mitigation technique at the receiver side, by using, such as, maximum likelihood detection, successive interference cancellation, multiple-antenna based interference nulling, etc. On the other hand, BS cooperation can be another efficient InterCI mitigation approach, which shifts the processing burden to the BSs, rather than causing too much computational complexity at mobile terminals $[16,19$, 20]. For example, the authors in $[19,20]$ have studied the scheduling and power-allocation in the context of the multicell downlink OFDMA systems and other networks, by handling the InterCI via BS coordination supported by the CSI exchange among BSs. By contrast, the researches in [10,21-23] have been devoted to the resource allocation in multicell OFDMA systems with full BS cooperation, which requires BSs to share both CSI and data. Under the constraint of certain backhaul capacity, a heuristic BS assignment algorithm has been proposed in [22], and a user scheduling algorithm has been developed in [23], respectively. Furthermore, in [24, 25], the authors have addressed the energy-efficiency issue of the BS cooperation based resource allocation in multicell OFDMA systems.

Against the background, in this paper, we investigate both the subcarrier-allocation and InterCI mitigation in multicell downlink OFDMA systems. In our considered systems, each cell independently allocates subcarriers based on our proposed bidirectional worst subchannel avoiding (BWSA) algorithm [26]. Our focus is on the InterCI mitigation after the distributed subcarrier-allocation. We propose two novel InterCI mitigation algorithms. The first one is the distributed decision making assisted cooperation (DDMC) algorithm, which motivates to maximize the pay-off of BS cooperation, while simultaneously minimize the caused cost. The second InterCI mitigation algorithm proposed is named as the centralized decision making assisted cooperation (CDMC), which motivates to make the best InterCI mitigation decisions based on the limited discrete InterCI information of the cell-edge users shared among the BSs, in order to maximize both the spectralefficiency, and the frequency reuse factor of the frequency spectrum. In this paper, we study and compare the spectralefficiency of cell-edge users, frequency reuse factor, overhead, etc., of the multicell downlink OFDMA systems employing the BWSA and various InterCI mitigation algorithms. Our studies and performance results show that both the proposed DDMC and CDMC algorithms are high-efficiency InterCI mitigation algorithms, which outperform the existing on-off power (OOP) algorithm in terms of the spectral-efficiency. The CDMC algorithm outperforms the DDMC algorithm, and is capable of achieving the sum rate close to the upperbound achieved by the full InterCI information assisted decision making (FIIDM) algorithm. In this FIIDM algorithm, cooperation decisions are made via the exhaustive search with ideal information about the InterCI. Additionally, the CDMC algorithm is demonstrated to have the highest frequency reuse factor in addition to its spectral-efficiency advantage.

The rest of this paper is organized as follows. Section II introduces the system model. Section III provides the gen-

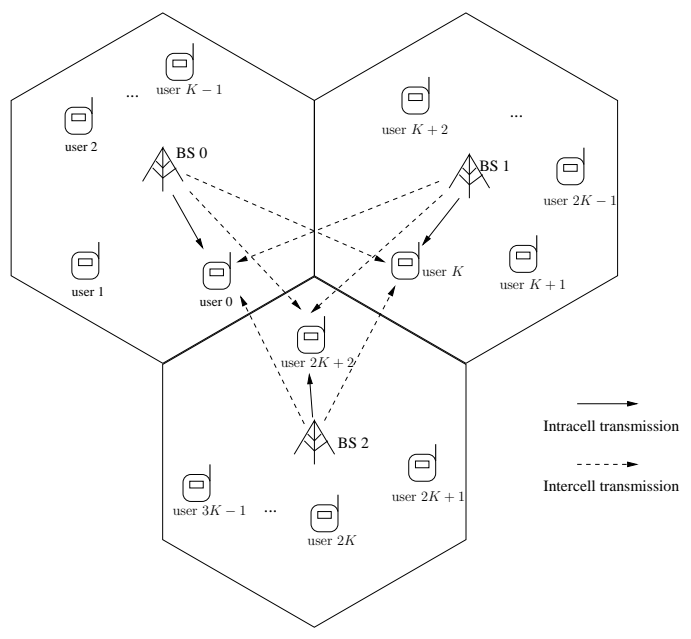

Fig. 1. Conceptual structure of the multicell downlink OFDMA systems.

eral theory about the distributed subcarrier-allocation and the InterCI mitigation. In Section IV, we discuss the FIIDM which is the upper-bound of our InterCI mitigation. Section V extends the OOP algorithm to the multicell downlink OFDMA systems. Sections VI and VII detail the proposed DDMC and CDMC algorithms, respectively. Performance results are shown in Section VIII. Finally, we summarize the main conclusions in Section IX.

\section{System Model}

In order to reflect the main features of multicell systems while make the problems relatively easy to mange, in this paper, we consider the same system model studied in [10,27-29], which is a three-cell downlink OFDMA system, as depicted in Fig. 1. In this system, each cell has one BS communicating with $K$ mobile users. Each of the communication terminals, including both BSs and mobile users, is assumed to employ one antenna for signal receiving and transmission. The BSs communicate with their users based on OFDMA having in total $M$ subcarriers.

We consider the extreme case that each cell supports $K=$ $M$ users and, hence, each user is assigned one subcarrier. Note that, we assume this extreme case for the sake of avoiding considering the trivial cases but focusing our attention on the InterCI mitigation. For the case where one user is assigned multiple subcarriers, the system can be modified to use our model by dividing one user into several ones of each assigned one subcarrier. However, in this case, the InterCI mitigation may become easier, owing to the reduced number of users involved. There is no IntraCI, since all users in one cell communicate on orthogonal subcarriers. However, without using InterCI mitigation, each user experiences InterCI from two users located respectively in the other two cells, which are assigned the same subcarrier as the considered user. Based on the above assumptions, therefore, the subcarrier-allocation 
should satisfy the constraints of

$$
\begin{aligned}
& \bigcup_{m \in \mathcal{M}} \mathcal{F}_{m}^{(u)}=\mathcal{K}^{(u)}, \forall u \in\{0,1,2\}, \\
& \mathcal{F}_{m}^{(u)} \bigcap \mathcal{F}_{m^{\prime}}^{(u)}=\emptyset, m \neq m^{\prime}, \forall m, m^{\prime} \in \mathcal{M}, \forall u \in\{0,1,2\},
\end{aligned}
$$

$$
\left|\mathcal{F}_{m}^{(u)}\right|=1, \forall m \in \mathcal{M}, \forall u \in\{0,1,2\}
$$

where $\mathcal{M}=\{0,1, \ldots, M-1\}$ is the set of subcarrier indexes, $\mathcal{F}_{m}^{(u)}$ contains the indexes of the users assigned to subcarrier $m$ in cell $u$, and $\mathcal{K}^{(u)}=\{u K+0, u K+1, \ldots, u K+K-1\}$ holds the indexes of the $K$ users in cell $u$. Note that, in the above equations, (1) explains that each BS assigns $M$ subcarriers to its $K$ users, while (2) and (3) impose the constraints that, in one cell, different users are allocated different subcarriers, and one user is assigned just one subcarrier.

As shown in Fig. 1, the BSs are located at the centers of the cells, and each cell has $K$ users, which are assumed to obey uniform distribution. In each of the three cells, we assume for simplicity the ideal power control as in [2,3,21,22], in order to maintain the same average received power of one unit per user. Furthermore, we assume that InterCI only exists between adjacent cells as the result of propagation pathloss. Let the InterCI be characterized by a factor $\alpha$. Then, when taking into account of the combined effect of propagation pathloss and shadowing, we can have [30]

$$
\alpha=\sqrt{\left(\frac{d_{0}}{d_{1}}\right)^{\mu} 10^{\frac{\zeta_{0}-\zeta_{1}}{10}}}
$$

where $d_{0}$ and $d_{1}$ represent the distances from a BS to the considered intracell and intercell users, respectively, $\mu$ is the pathloss exponent, while $\zeta_{0}$ and $\zeta_{1}$ (in $\mathrm{dB}$ ) are the zero-mean Gaussian random variables with a standard deviation $\Upsilon$ (in $\mathrm{dB})$, which account for the shadowing effect [30]. In addition to the propagation pathloss and shadowing effects, signals transmitted from BSs also experience fast fading, which is assumed to be the independent Rayleigh flat fading in terms of different users.

Let us assume that a data symbol to be transmitted by BS $u$ to its intracell user $k\left(k \in \mathcal{K}^{(u)}\right)$ is expressed as $x_{k}^{(u)}$, which satisfies $E\left[x_{k}^{(u)}\right]=0$ and $E\left[\left|x_{k}^{(u)}\right|^{2}\right]=1$. Since the $M$ subcarriers in one cell are assumed to be orthogonal, the signal received by user $k$ of cell $u$ can be written as

$$
\begin{aligned}
& y_{k}^{(u)}=h_{k, m}^{(u)} w_{k, m}^{(u)} x_{k}^{(u)}+ \\
& \underbrace{h_{k, m}^{\left(u^{\prime}\right)} \alpha_{k^{\prime}, k}^{\left(u^{\prime}\right)} w_{k^{\prime}, m}^{\left(u^{\prime}\right)} x_{k^{\prime}}^{\left(u^{\prime}\right)}+h_{k, m}^{\left(u^{\prime \prime}\right)} \alpha_{k^{\prime \prime}, k}^{\left(u^{\prime \prime}\right)} w_{k^{\prime \prime}, m}^{\left(u^{\prime \prime}\right)} x_{k^{\prime \prime}}^{\left(u^{\prime \prime}\right)}}_{\text {InterCI }}+n_{k}^{(u)}
\end{aligned}
$$

when assuming that $k \in \mathcal{F}_{m}^{(u)}, k^{\prime} \in \mathcal{F}_{m}^{\left(u^{\prime}\right)}$ and $k^{\prime \prime} \in \mathcal{F}_{m}^{\left(u^{\prime \prime}\right)}$, meaning that users $k, k^{\prime}$ and $k^{\prime \prime}$ in cells $u, u^{\prime}$ and $u^{\prime \prime}$, respectively, are assigned to share subcarrier $m$. Hence, users $k, k^{\prime}$ and $k^{\prime \prime}$ are referred to as the co-subcarrier users. In (5), $n_{k}^{(u)}$ represents the Gaussian noise at user $k$, which is assumed to obey the complex Gaussian distribution with zero mean and a variance of $2 \sigma^{2}=1 / \gamma_{s}$, where $\gamma_{s}$ denotes the average SNR per symbol. $h_{k, m}^{(u)}$ denotes the fast fading gain on the $m$ th subcarrier from BS $u$ to user $k$, and $h_{k, m}^{\left(u^{\prime}\right)} \alpha_{k^{\prime}, k}^{\left(u^{\prime}\right)}$ represents the
InterCI that user $k$ receives from $\mathrm{BS} u^{\prime}$, when it uses subcarrier $m$ to send signals to user $k^{\prime}$. Here, $h_{k, m}^{\left(u^{\prime}\right)}$ is the fast fading gain on the $m$ th subcarrier from BS $u^{\prime}$ to user $k$, and $\alpha_{k^{\prime}, k}^{\left(u^{\prime}\right)}$ is the corresponding InterCI factor. In this paper, we assume that the uplinks and downlinks are operated in the TDD mode, and a BS is capable of acquiring the CSI of the channels between the BS and its $K$ intracell users. In this case, a BS is capable of preprocessing the signals to be transmitted to its intracell users by setting $w_{k, m}^{(u)}$ seen in (5) as $w_{k, m}^{(u)}=\frac{\left(h_{k, m}^{(u)}\right)^{*}}{\sqrt{\left|h_{k, m}^{(u)}\right|^{2}}}$, where $(\cdot)^{*}$ denotes the conjugate operation. We assume that any BS does not have the CSI of the InterCI channels, including both the slow and fast fading, which is possibly due to the complexity constraint. From (5), the signal-to-interference-plus-noise ratio (SINR) for user $k$ can be expressed as

$$
\begin{aligned}
\gamma_{k, m}^{(u)} & =\frac{\left|h_{k, m}^{(u)}\right|^{2}}{\left|h_{k, m}^{\left(u^{\prime}\right)} \alpha_{k^{\prime}, k}^{\left(u^{\prime}\right)}\right|^{2}+\left|h_{k, m}^{\left(u^{\prime \prime}\right)} \alpha_{k^{\prime \prime}, k}^{\left(u^{\prime \prime}\right)}\right|^{2}+2 \sigma^{2}} \\
& =\frac{\left|h_{k, m}^{(u)}\right|^{2}}{I_{u^{\prime}, k}+I_{u^{\prime \prime}, k}+2 \sigma^{2}}, m \in \mathcal{M}
\end{aligned}
$$

where $I_{u^{\prime}, k}=\left|h_{k, m}^{\left(u^{\prime}\right)} \alpha_{k^{\prime}, k}^{\left(u^{\prime}\right)}\right|^{2}$ is the InterCI power received by user $k$ from BS $u^{\prime}$. Alternatively, (6) can be written as

$$
\begin{aligned}
\gamma_{k, m}^{(u)} & =\frac{1}{\left(\eta_{k, m}^{(u)}\right)^{-1}+\left(A_{k, m}^{(u)}\right)^{-1}}, \\
\eta_{k, m}^{(u)} & =\frac{\left|h_{k, m}^{(u)}\right|^{2}}{I_{u^{\prime}, k}+I_{u^{\prime \prime}, k}}, A_{k, m}^{(u)}=\frac{\left|h_{k, m}^{(u)}\right|^{2}}{2 \sigma^{2}}
\end{aligned}
$$

where $\eta_{k, m}^{(u)}$ and $A_{k, m}^{(u)}$ are respectively the signal-tointerference ratio (SIR) and signal-to-noise ratio (SNR) of user $k$ in cell $u$.

From (6) and (7) we imply that, in order to achieve high SINR but at low implementation complexity, we may design the subcarrier-allocation motivating to maximize the channel gains from a BS to its $K$ intercell users, while the InterCI mitigation aiming to minimize the InterCI with the backhaul cost as low as possible. For these purposes, we consider two InterCI mitigation methods, which are the power off and $B S$ cooperation. With the power off method, the transmissions to some users experiencing strong InterCI are turned off. The method is easy to operate, does not require BS cooperation and is sometimes very efficient, as shown in [31].

By contrast, when the BS cooperation method is employed, we assume that a mobile user can estimate the strength of the signal from its own BS and the power of the InterCI signals from the two interfering BSs. As the BS cooperation motivates to rely on the lowest possible backhaul cost, we assume that there is no CSI sharing among the BSs. In this case, a promising BS cooperation scheme is the classic space time block coding (STBC) [32], which only needs to exchange the data symbols of the users requiring BS cooperation. Consequently, when two BSs use, such as, Alamouti's STBC [32] to send information to one user, two orders of transmit diversity can be achieved. In this way, we may enhance the detection reliability and/or the throughput to the system, in comparison with the power off scheme. Let us illustrate this following 
(5). Let us assume that BS $u^{\prime}$ cooperating with BS $u$ to transmit $x_{k}^{(u)}(t)$ and $x_{k}^{(u)}(t+T)$ to user $k$ based on Alamouti's scheme [32], where $T$ represents the symbol duration. Then, the observations received by user $k$ at time $t$ and $t+T$ can be written as

$$
\begin{aligned}
y_{k}^{(u)}(t)= & h_{k, m}^{(u)} x_{k}^{(u)}(t)+h_{k, m}^{\left(u^{\prime}\right)} \alpha_{k^{\prime}, k}^{\left(u^{\prime}\right)} x_{k}^{(u)}(t+T)+ \\
& h_{k, m}^{\left(u^{\prime \prime}\right)} \alpha_{k^{\prime \prime}, k}^{\left(u^{\prime \prime}\right)} x_{k^{\prime \prime}}^{\left(u^{\prime \prime}\right)}(t)+n_{k}^{(u)}(t), \\
y_{k}^{(u)}(t+T)= & -h_{k, m}^{(u)}\left(x_{k}^{(u)}(t+T)\right)^{*}+h_{k, m}^{\left(u^{\prime}\right)} \alpha_{k^{\prime}, k}^{\left(u^{\prime}\right)}\left(x_{k}^{(u)}(t)\right)^{*}+ \\
& h_{k, m}^{\left(u^{\prime \prime}\right)} \alpha_{k^{\prime \prime}, k}^{\left(u^{\prime \prime}\right)} x_{k^{\prime \prime}}^{\left(u^{\prime \prime}\right)}(t+T)+n_{k}^{(u)}(t+T) .
\end{aligned}
$$

Assume that user $k$ is capable of estimating the channels from BSs $u$ and $u^{\prime}$. Then, it can form the decision variables for detecting $x_{k}^{(u)}(t)$ and $x_{k}^{(u)}(t+T)$ as

$$
\begin{aligned}
& r_{k}^{(u)}(t)=\left(h_{k, m}^{(u)}\right)^{*} y_{k}^{(u)}(t)+h_{k, m}^{\left(u^{\prime}\right)} \alpha_{k^{\prime}, k}^{\left(u^{\prime}\right)}\left(y_{k}^{(u)}(t+T)\right)^{*}, \quad \\
& r_{k}^{(u)}(t+T)=\left(h_{k, m}^{\left(u^{\prime}\right)} \alpha_{k^{\prime}, k}^{\left(u^{\prime}\right)}\right)^{*} y_{k}^{(u)}(t)-h_{k, m}^{(u)}\left(y_{k}^{(u)}(t+T)\right)^{*} .
\end{aligned}
$$

From (10) and (11), we can derive the SINR of user $k$ for detecting $x_{k}(t)$ and $x_{k}(t+T)$, which is

$$
\gamma_{k}^{(u)}=\frac{\left|h_{k, m}^{(u)}\right|^{2}+\left|h_{k, m}^{\left(u^{\prime}\right)} \alpha_{k^{\prime}, k}^{\left(u^{\prime}\right)}\right|^{2}}{\left|h_{k, m}^{\left(u^{\prime \prime}\right)} \alpha_{k^{\prime \prime}, k}^{\left(u^{\prime \prime}\right)}\right|^{2}+2 \sigma^{2}} .
$$

Note that, the above cooperation is usually set up, when BS $u^{\prime}$ generates strong InterCI on user $k$, meaning that the term of $\left|h_{k, m}^{\left(u^{\prime}\right)} \alpha_{k^{\prime}, k}^{\left(u^{\prime}\right)}\right|^{2}$ in the above equation has a relatively large value. In this case, the SINR of (12) resulted from the cooperation can be significantly enhanced in comparison with the SINR of (6) of the case without BS cooperation, which consequently improves the multicell system's overall throughput.

\section{General THEORY}

In this section, we address the general theory of the distributed subcarrier-allocation and the design motivation for the InterCI mitigation in the multicell downlink OFDMA systems. For achieving relatively low-complexity implementation, in this paper we propose to first carry out the distributed subcarrier-allocation, and then operate the InterCI mitigation, when different levels of BS cooperation are considered. The distributed subcarrier-allocation is motivated to maximize the sum rate of each cell, with the optimization problem described as

$$
\begin{aligned}
\left\{\mathcal{F}_{m}^{(u)}, \forall m\right\}^{*}= & \arg \max _{\left\{\mathcal{F}_{m}^{(u)}, \forall m\right\}}\left\{\sum_{k \in \mathcal{K}^{(u)}} \log _{2}\left(1+\gamma_{k}^{(u)}\right)\right\} \\
& \forall u \in\{0,1,2\} \\
& \text { subject to (1), (2), (3) }
\end{aligned}
$$

where $\gamma_{k}^{(u)}$ is the SINR of user $k$ in cell $u$, such as that in (6). In (13), $\left\{\mathcal{F}_{m}^{(u)}, \forall m\right\}$ means testing all the possible subcarrierallocations for cell $u$, while $\left\{\mathcal{F}_{m}^{(u)}, \forall m\right\}^{*}$ returns the final results of the subcarrier-allocation.

However, the problem in (13) is a mixed integer nonconvex problem that is very hard to solve. Therefore, as done in [6,
26,33 ], the distributed subcarrier-allocation can be motivated to maximize the SNRs of all the users in one cell without considering the impact of InterCI. Correspondingly, this optimization problem can be expressed as

$$
\begin{aligned}
\left\{\mathcal{F}_{m}^{(u)}, \forall m\right\}^{*}= & \arg \max _{\left\{\mathcal{F}_{m}^{(u)}, \forall m\right\}}\left\{A_{k}^{(u)}, k \in \mathcal{K}^{(u)}\right\}, \forall u \in\{0,1,2\} \\
& \text { subject to (1), (2), (3) }
\end{aligned}
$$

where $A_{k}^{(u)}$ is the SNR of user $k$, such as that defined in (7). Based on (14), in [26], we have designed a bidirectional worst subchannel avoiding (BWSA) algorithm for the single-cell OFDMA systems, which is demonstrated to be low-complexity and capable of achieving near-optimum performance. In this paper, we investigate the performance of the multicell downlink OFDMA systems employing the BWSA algorithm in association with our proposed and other InterCI mitigation algorithms.

As the subcarrier-allocation considered above does not deal with the InterCI, after the subcarrier-allocation, the InterCI mitigation is then operated for the cell-edge users. Let us define the user set of cell $u$ as $\hat{\mathcal{K}}^{(u)}=\left\{k \mid \eta_{k}<\eta_{t}, k \in \mathcal{K}^{(u)}\right\}$, where $\eta_{t}$ represents a SIR threshold. The threshold $\eta_{t}$ can be set according to various communication objectives. Then, the users in set $\tilde{\mathcal{K}}^{(u)}$ are called as the cell-edge users of cell $u$. Here, the set $\tilde{\mathcal{K}}^{(u)}$ includes both the users in $\hat{\mathcal{K}}^{(u)}$ as well as the users in $\mathcal{K}^{(u)}-\hat{\mathcal{K}}^{(u)}$ that share the same subcarriers as the users in $\hat{\mathcal{K}}^{\left(u^{\prime}\right)}$ of cell $u^{\prime}$ and the users in $\hat{\mathcal{K}}^{\left(u^{\prime \prime}\right)}$ of cell $u^{\prime \prime}$. In general, our InterCI mitigation motivates to maximize the sum rate of the cell-edge users by solving the optimization problem of

$\boldsymbol{D}^{*}=\arg \max _{\boldsymbol{D}}\left\{\sum_{u=0}^{2} \sum_{k \in \tilde{\mathcal{K}}^{(u)}} \log _{2}\left(1+\gamma_{k}^{(u)}\right) \mid\left\{\mathcal{F}_{m}^{(u)}, \forall m, u\right\}^{*}\right\}$

where $3 M$-length InterCI mitigation decision (IMD) vector can be written in the form of $\boldsymbol{D}=\left[\boldsymbol{D}_{0}^{T}, \ldots, \boldsymbol{D}_{M-1}^{T}\right]^{T}$, where $(\cdot)^{T}$ is the transpose operation. Here, $\boldsymbol{D}_{m}=$ $\left[D_{0, m}, D_{1, m}, D_{2, m}\right]^{T}$ is referred to as the IMD vector of subcarrier $m$, which defines the transmission states of the users in the three cells assigned subcarrier $m$.

In order to minimize the cost of backhaul resources for BS cooperation, in this paper, we classify $D_{u, m}$ only into three states. Let us again assume that subcarrier $m$ is assigned to user $k, k^{\prime}$ and $k^{\prime \prime}$ in cell $u, u^{\prime}$ and $u^{\prime \prime}$, respectively. Then, the three states of $D_{u, m}$ are defined as

$$
D_{u, m}= \begin{cases}k & \text { BS } u \text { transmits } x_{k}^{(u)} \text { to its intracell } \\ & \text { user } k \text { on subcarrier } m, \\ & \text { BS } u \text { switches off its transmission } \\ & \text { on subcarrier } m, \\ k^{\prime}\left(\text { or } k^{\prime \prime}\right) \quad & \text { BS } u \text { cooperates to transmit } x_{k^{\prime}}^{\left(u^{\prime}\right)} \\ & \left.\left(\text { or } x_{k^{\prime \prime}}^{\left(u^{\prime \prime}\right)}\right) \text { to user } k^{\prime} \text { (or } k^{\prime \prime}\right) \text { in cell } \\ & u^{\prime}\left(\text { or } u^{\prime \prime}\right) \text { on subcarrier } m .\end{cases}
$$


Correspondingly, the InterCI mitigation is carried out under the constraints of

$$
\begin{aligned}
& D_{u, m} \in\left\{k, k^{\prime}, k^{\prime \prime},-1\right\}, \\
& \sum_{u=0}^{2} D_{u, m} \geq-2
\end{aligned}
$$

for $u \in\{0,1,2\}$ and $m \in \mathcal{M}$. Note that, the constraint of (18) prevents from switching off all the three transmissions on one subcarrier. Furthermore, as shown in Section II, InterCI only exists among the three users sharing a subcarrier. Therefore, the InterCI mitigation can be considered subcarrierby-subcarrier independently without performance loss. Hence, by considering the constraints of (17) and (18), we can re-write the optimization problem of (15) as

$$
\begin{aligned}
\boldsymbol{D}_{m}^{*}= & \underset{\arg \max _{\boldsymbol{D}_{m}}}{ }\left\{\sum_{u=0}^{2} \log _{2}\left(1+\gamma_{k}^{(u)}\right), k \in \tilde{\mathcal{K}}^{(u)} \cap \mathcal{F}_{m}^{(u)} \mid\left\{\mathcal{F}_{m}^{(u)}, \forall u\right\}^{*}\right\}, \\
& \forall m \in \mathcal{M} \\
& \text { subject to (17) and (18). }
\end{aligned}
$$

It can be shown that both (15) and (19) are the mixed integer nonlinear nonconvex problems, whose optimal solutions are extremely hard to derive. Below we will propose two novel InterCI mitigation algorithms, namely the DDMC and CDMC, which aim to find the promising sub-optimal solutions for the problem of (19). Furthermore, we extend the OOP algorithm $[31,34,35]$ to the multicell downlink OFDMA systems, and investigate its performance in association with the BWSA subcarrier-allocation. Additionally, as a benchmark, we also consider the FIIDM scheme, which uses exhaustive search to find the optimal solutions for (19).

\section{Full INTERCI INFORMATION RELIED DECISION MAKING ALGORITHM}

As above-mentioned, the OOP, DDMC and the CDMC algorithms will be compared against the FIIDM algorithm, which relies on the continuous InterCI information, in contrast to the discrete InterCI information used by the DDMC and CDMC algorithms. Furthermore, the FIIDM algorithm uses exhaustive search to find the optimum solutions to the problem of (19). Hence, its performance represents an upper-bound of the InterCI mitigation algorithms considered. The FIIDM algorithm can be described by Algorithm 1 with the aid of some further explanation.

\section{Algorithm 1: (FIIDM Algorithm)}

\section{Initialization:}

(1) Set $\tilde{\mathcal{K}}_{m}=\left\{k \mid k \in \tilde{K}^{(u)} \cap \mathcal{F}_{m}^{(u)}, \forall u \in\{0,1,2\}\right\}, \forall m \in$ $\mathcal{M}$.

(2) Set $D_{u, m}=k$ if $\mathcal{F}_{m}^{(u)}=\{k\}, \forall u \in\{0,1,2\}, \forall m \in \mathcal{M}$.

For Subcarrier $m \in \mathcal{M}$ :

If $\tilde{\mathcal{K}}_{m} \neq \emptyset$, the central unit (CU) first collects the InterCI information of all the users in $\tilde{\mathcal{K}}_{m}$, and then executes:

Step 1 Compute the sum rates of all the optional decisions with power off only. The optional decisions include: (1) Power off to one user: $\hat{D}_{u, m}=-1, \hat{D}_{u^{\prime}, m}=$
$D_{u^{\prime}, m}, \hat{D}_{u^{\prime \prime}, m}=D_{u^{\prime \prime}, m}, \forall u, u^{\prime}, u^{\prime \prime} \in\{0,1,2\}$ and $u \neq u^{\prime} \neq u^{\prime \prime}$.

(2) Power off to two users: $\hat{D}_{u, m}=\hat{D}_{u^{\prime}, m}=-1$, $\hat{D}_{u^{\prime \prime}, m}=D_{u^{\prime \prime}, m}, \forall u, u^{\prime}, u^{\prime \prime} \in\{0,1,2\}$ and $u \neq$ $u^{\prime} \neq u^{\prime \prime}$

Step 2 Compute the sum rates of all the optional decisions with cooperation only. The optional decisions include:

(1) Cooperation between two BSs: $\hat{D}_{u, m}=\hat{D}_{u^{\prime}, m}=$ $D_{u, m}, \hat{D}_{u^{\prime \prime}, m}=D_{u^{\prime \prime}, m}, \forall u, u^{\prime}, u^{\prime \prime} \in\{0,1,2\}$ and $u \neq u^{\prime} \neq u^{\prime \prime}$.

(2) Cooperation among three BSs: $\hat{D}_{u, m}=\hat{D}_{u^{\prime}, m}=$ $\hat{D}_{u^{\prime \prime}, m}=D_{u, m}, \forall u, u^{\prime}, u^{\prime \prime} \in\{0,1,2\}$ and $u \neq u^{\prime} \neq$ $u^{\prime \prime}$.

Step 3 Compute the sum rates of all the optional decisions with power off and/or cooperation. The optional decisions include:

(1) One BS sets power off to one user while the other two BSs cooperate for one user: $\hat{D}_{u, m}=\hat{D}_{u^{\prime}, m}=$ $D_{u, m}, \hat{D}_{u^{\prime \prime}, m}=-1, \forall u, u^{\prime}, u^{\prime \prime} \in\{0,1,2\}$ and $u \neq u^{\prime} \neq u^{\prime \prime}$.

Step 5 The CU first identifies the best one among the above optional decisions, which can be expressed as:

$$
\boldsymbol{D}_{m}=\arg \max _{\hat{\boldsymbol{D}}_{m}}\left\{\sum_{k \in \tilde{\mathcal{K}}_{m}} \log _{2}\left(1+\gamma_{k}\right)\right\}
$$

where $\hat{\boldsymbol{D}}_{m}=\left[\hat{D}_{u, m}, \hat{D}_{u^{\prime}, m}, \hat{D}_{u^{\prime \prime}, m}\right]^{T}$. Then, the CU informs the final IMD vector $\boldsymbol{D}_{m}=$ $\left[D_{u, m}, D_{u^{\prime}, m}, D_{u^{\prime \prime}, m}\right]^{T}$ to the three BSs.

As shown in Algorithm 1, the FIIDM algorithm assumes that there is a CU, which is capable of collecting the ideal continuous InterCI information of all the cell-edge users. Based on the InterCI information collected, the CU then makes the optimum InterCI mitigation decisions by exhaustive search, and finally informs them to the BSs. From Algorithm 1, we can find that, there are in total 21 optional decisions for one cell-edge user group, such as that in $\tilde{\mathcal{K}}_{m}$, containing three cosubcarrier users. Specifically, at Step 2, the FIIDM algorithm may turn off one or two transmissions to the three users, which gives 6 optional decisions. At Step 3, any one or two BSs may help another BS to set up a cooperative transmission, which gives 9 different decisions. Finally, at Step 4, two BSs may cooperate while the other one is turned off, resulting in total 6 optional decisions. Therefore, there are in total 21 optional decisions. In Algorithm 1, (20) finds the best one among these 21 optional decisions.

From Algorithm 1 and the above analysis, we know that for the three-cell OFDMA systems, the decision making process of the FIIDM algorithm does not impose much complexity. As for each $\tilde{\mathcal{K}}_{m}$, there are only three co-subcarrier users resulting in 21 optional decisions to be considered. However, the algorithm requires the continuous InterCI information of the cell-edge users for decision making, which may be sent to a CU or shared by the three BSs. This process may impose a heavy complexity burden on the backhaul network, especially, when there is a big number of the cell-edge users. Furthermore, 
it may be very hard to implement the FIIDM algorithm in the practical scenarios having a large number of cells. Therefore, we propose the more practical DDMC and CDMC algorithms, which only require the limited discrete InterCI information.

\section{ON-OfF Power InterCi Mitigation}

The OOP algorithm employs an efficient method to combat InterCI, which does not require BS cooperation. It has been widely studied and used in multicell communication systems, such as, in $[31,34,35]$. The basic principle of the OOP algorithm is to allow a BS to turn off the transmission on the subchannels conflicting strong InterCI. By doing this, there are two-fold of benefits. First, transmission on the poor subchannels can be avoided, which saves power for the future transmission, when the subchannels become better. Second, the InterCI imposed by these subchannels on the other cells can also be removed. The OOP algorithm is usually scheduled to be carried out by a BS at a time, in order to avoid that two or three cells simultaneously turn off the transmission on the same subcarrier.

Let us below illustrate the OOP algorithm with the aid of an example. Assume that subcarrier $m$ is allocated to users $k, k^{\prime}$, $k^{\prime \prime}$ in cells $u, u^{\prime}$ and $u^{\prime \prime}$, respectively. Then, we can express the subchannel qualities on subcarrier $m$ in a matrix form as

$$
\begin{aligned}
& \boldsymbol{A}_{m}=\left[\begin{array}{ccc}
A_{k, m}^{(u)} & A_{k^{\prime}, m}^{(u)} & A_{k^{\prime \prime}, m}^{(u)} \\
A_{k, m}^{\left(u^{\prime}\right)} & A_{k^{\prime}, m}^{\left(u^{\prime}\right)} & A_{k^{\prime \prime}, m}^{\left(u^{\prime}\right)} \\
A_{k, m}^{\left(u^{\prime \prime}\right)} & A_{k^{\prime}, m}^{\left(u^{\prime \prime}\right)} & A_{k^{\prime \prime}, m}^{\left(u^{\prime \prime}\right)}
\end{array}\right] \\
& =\left[\begin{array}{ccc}
\frac{\left|h_{k, m}^{(u)}\right|^{2}}{2 \sigma^{2}} & \frac{\left|h_{k^{\prime}, m}^{(u)} \alpha_{k, k^{\prime}}^{(u)}\right|^{2}}{2 \sigma^{2}} & \frac{\left|h_{k^{\prime \prime}, m}^{(u)} \alpha_{k, k^{\prime \prime}}^{(u)}\right|^{2}}{2 \sigma^{2}} \\
\frac{\left|h_{k, m}^{\left(u^{\prime}\right)} \alpha_{k^{\prime}, k}^{\left(u^{\prime}\right)}\right|^{2}}{2 \sigma^{2}} & \frac{\left|h_{k^{\prime}, m}^{\left(u^{\prime}\right)}\right|^{2}}{2 \sigma^{2}} & \frac{\left|h_{k^{\prime \prime}, m}^{\left(u^{\prime}\right)} \alpha_{k^{\prime}, k^{\prime \prime}}^{\left(u^{\prime}\right)}\right|^{2}}{2 \sigma^{2}} \\
\frac{\left|h_{k, m}^{\left(u^{\prime \prime}\right)} \alpha_{k^{\prime \prime}, k}^{2}\right|^{\prime \prime}}{2 \sigma^{2}} & \frac{\mid h_{k^{\prime}, m}^{\left(u^{\prime \prime}\right)} \alpha_{k^{\prime \prime}, k^{\prime}}^{\left(u^{\prime \prime}\right)}}{2 \sigma^{2}} & \frac{\left|h_{k^{\prime \prime}, m}^{\left(u^{\prime \prime}\right)}\right|^{2}}{2 \sigma^{2}}
\end{array}\right]
\end{aligned}
$$

where $A_{j, m}^{(i)}$ represents the subchannel quality of the transmission from BS $i$ to user $j$ on subcarrier $m$. Based on a column of $A_{m}$, we can calculate a user's SIR. For example, the SIR of user $k$ is given by $\eta_{k, m}^{(u)}=\frac{A_{k, m}^{(u)}}{A_{k, m}^{\left(u^{\prime}\right)}+A_{k, m}^{\left(u^{\prime \prime}\right)}}$.

Let us consider one realization of the above example, and the matrix is given by

$$
A_{m}=\left[\begin{array}{lll}
2.1909 & 0.0018 & 0.5078 \\
1.4294 & 1.8621 & 0.1583 \\
0.1168 & 3.3187 & 1.6459
\end{array}\right]
$$

Then, by setting different SIR thresholds, the OOP algorithm generates different results for the IMD vectors $D_{m}$, and derives different sum rates $C_{\Sigma}=\sum_{i \in\left\{k, k^{\prime}, k^{\prime \prime}\right\}} \log _{2}\left(1+\gamma_{i}\right)$. Note that, for the example we assume the unit noise power. Specifically, for (22), when the SIR thresholds are respectively $\eta_{t}=-5 d B, 0 d B$ and $5 d B$, the OOP algorithm gives the InterCI mitigation decisions as

$$
\left\{\begin{array}{l}
\text { (a): } D_{u, m}=k, D_{u^{\prime}, m}=k^{\prime}, D_{u^{\prime \prime}, m}=k^{\prime \prime} \text { if } \eta_{t}=-5 \mathrm{~dB}, \\
\text { (b): } D_{u, m}=k, D_{u^{\prime}, m}=-1, D_{u^{\prime \prime}, m}=k^{\prime \prime} \text { if } \eta_{t}=0 \mathrm{~dB}, \\
\text { (c): } D_{u, m}=D_{u^{\prime}, m}=-1, D_{u^{\prime \prime}, m}=k^{\prime \prime} \text { if } \eta_{t}=5 \mathrm{~dB}
\end{array}\right.
$$

which are explained as follows. First, if $\eta_{t}=-5 \mathrm{~dB}=0.316$, there is no user turned off, since the SIRs of the three users are all higher than this SIR threshold. In this case, the sum rate on subcarrier $m$ is $C_{\Sigma}=2.4039$. Second, when $\eta_{t}=0 \mathrm{~dB}=1$, during the first stage, user $k$ stays on, since its SIR is $\eta_{k, m}^{(u)}=$ $1.4171>\eta_{t}$. During the second stage, the transmission to user $k^{\prime}$ is switched off, as its SIR of $\eta_{k^{\prime}, m}^{\left(u^{\prime}\right)}=0.5608$ is lower than the threshold. During the third stage, user $k^{\prime \prime}$ finds that its SIR is higher than the threshold, after user $k^{\prime \prime}$ is turned off. Hence, it stays on. In this case, the sum rate becomes $C_{\Sigma}=2.6311$, which is higher than $C_{\Sigma}=2.4039$ of the first case. Finally, when $\eta_{t}=5 \mathrm{~dB}=3.1623$, the OOP algorithm turns off the transmissions to users $k$ and $k^{\prime \prime}$. In this case, the sum rate attained on subcarrier $m$ is $C_{\Sigma}=1.4038$, which is also lower than that obtained in the case of $\eta_{t}=0 \mathrm{~dB}$.

From the above example, we know that the performance of the system employing the OOP algorithm is highly dependent on the SIR threshold. If an improper SIR threshold is set, it may turn off too many or too few subchannels, which may lead to the degradation of throughput performance.

\section{Distributed Decision Making Assisted COOPERATION INTERCI Mitigation}

In this section, we propose a novel InterCI mitigation scheme referred to as the distributed decision making assisted cooperation (DDMC). As its name suggests, the DDMC algorithm introduces BS cooperation to improve the system performance. In Section III, we have shown the benefits from the cooperative transmission to a user, if the cooperative BS imposes strong InterCI on the user. However, the cost for this cooperation is the increase of the complexity for information exchange between the BSs, and the cooperative BS has to stop transmitting information to its own user. Therefore, our DDMC algorithm is motivated to maximize the pay-off from cooperation, while simultaneously minimize the cost caused by cooperation.

In the DDMC algorithm, the BSs are scheduled to make their InterCI mitigation decisions successively and independently. When the SIR measured by a user is lower than the SIR threshold, it informs its BS to take one of the two actions: 1) setting up a cooperative transmission for the user, and 2) switching off the transmission to the user. Let us below use the example shown in (21) to explain the principles. Assume that, the SIR of user $k$ is lower than the threshold $\eta_{t}$, the rules for user $k$ to choose the desired action are:

$$
\begin{aligned}
& \text { Cooperation from BS } u^{\prime} \text {, if } I_{u^{\prime}, k}>I_{c} \& I_{u^{\prime \prime}, k} \leq I_{c} \text {, } \\
& \text { Cooperation from BS } u^{\prime \prime} \text {, if } I_{u^{\prime}, k} \leq I_{c} \& I_{u^{\prime \prime}, k}>I_{c} \text {, } \\
& \text { Power off, if } I_{u^{\prime}, k}>I_{c} \& I_{u^{\prime \prime}, k}>I_{c} \\
& \qquad \text { or } I_{u^{\prime}, k} \leq I_{c} \& I_{u^{\prime \prime}, k} \leq I_{c} .
\end{aligned}
$$

Above, $I_{c}$ is the cooperation threshold, which can be set according to the various communication objectives, such as, maximization of sum rate. Note that, a user can only ask for cooperation when there is only one strong InterCI.

Let us now explain in detail why the rules in (24)-(26) are introduced with the aid of the example considered. First, 
suppose user $k$ obtains the cooperation from BS $u^{\prime}$, then, the SINRs of users $k, k^{\prime}$ and $k^{\prime \prime}$ become

$$
\begin{aligned}
\gamma_{k, m}^{(u)} & =\frac{\left|h_{k, m}^{(u)}\right|^{2}+I_{u^{\prime}, k}}{I_{u^{\prime \prime}, k}+2 \sigma^{2}}, \gamma_{k^{\prime}, m}^{\left(u^{\prime}\right)}=0, \\
\gamma_{k^{\prime \prime}, m}^{\left(u^{\prime \prime}\right)} & =\frac{\left|h_{k^{\prime \prime}, m}^{\left(u^{\prime \prime}\right)}\right|^{2}}{I_{u, k^{\prime \prime}}+I_{u^{\prime}, k^{\prime \prime}}+2 \sigma^{2}} .
\end{aligned}
$$

From (27), we can know that the SINR of user $k$ can be significantly improved, as the conditions in (24) are met. In this case, the sum rate of the three users is most probably increased, owing to making use of the strong InterCI of $I_{u^{\prime}, k}$. By contrast, when the conditions in (26) are met, we can know from (27) that the sum rate contributed by BS cooperation is insignificant. In these cases, it is better to simply turn off the transmission to user $k$, while keeping the other two users active.

In more detail, let us consider the values given in (22), from which we can find that the SIRs of the three uses are $\eta_{k, m}^{(u)}=$ 1.417, $\eta_{k^{\prime}, m}^{\left(u^{\prime}\right)}=0.5608$ and $\eta_{k^{\prime \prime}, m}^{\left(u^{\prime \prime}\right)}=2.471$, respectively. By setting the various SIR thresholds and InterCI thresholds for cooperation, the DDMC algorithm yields the IMD variables as

$\left\{\begin{array}{l}\text { (a): } D_{u, m}=k, D_{u^{\prime}, m}=D_{u^{\prime \prime}, m}=k^{\prime} \text { if } \eta_{t}=0 \mathrm{~dB}, I_{c}=1, \\ \text { (b): } D_{u, m}=D_{u^{\prime}, m}=k, D_{u^{\prime \prime}, m}=-1 \text { if } \eta_{t}=5 \mathrm{~dB}, I_{c}=1\end{array}\right.$

Let us first consider the case of (28)(a). In this case, user $k$ stays on during the first stage, as its SIR is higher than $\eta_{t}$. During the second stage, user $k^{\prime}$ finds that its SIR is lower than $\eta_{t}$. Then, it informs BS $u^{\prime}$ to request the cooperation from BS $u^{\prime \prime}$, since $I_{u, k^{\prime}} \leq I_{c}$ and $I_{u^{\prime \prime}, k^{\prime}}>I_{c}$, and the conditions in (26) are met. As a result, BS $u^{\prime \prime}$ switches off its transmission to user $k^{\prime \prime}$, and helps to transmit information to user $k^{\prime}$. Consequently, the sum rate of subcarrier $m$ is $C_{\Sigma}=3.5213$, which is higher than that achieved by the OOP algorithm. Similarly, in the case of (28)(b), the DDMC algorithm obtains the decision: BS $u$ obtains the cooperation from BS $u^{\prime}$ for user $k$, while BS $u^{\prime \prime}$ turns off the transmission to user $k^{\prime \prime}$. Consequently, the sum rate achieved is $C_{\Sigma}=2.2080$. Clearly, the sum rate is higher than 1.4038 obtained by the OOP algorithm for the corresponding case.

Based on the above analysis and the examples, we can now summarize the DDMC algorithm as follows.

Algorithm 2: (DDMCAlgorithm)

For Stage $u=0,1,2$ :

For User $k \in \mathcal{K}^{(u)}$ :

Initialization: Set $D_{u, m}=k$ if $\mathcal{F}_{m}^{(u)}=\{k\}, m \in \mathcal{M}$.

User $k$ estimates its SIR $\eta_{k, m}^{(u)}$. If $\eta_{k}<\eta_{t}$, execute:

Step 1 User $k$ informs BS $u$ the requirement of InterCI mitigation. Go to Step 2 if (26) is met, otherwise go to Step 3.

Step 2 BS $u$ switches off the transmission to user $k$, yielding $D_{u, m}=-1$.

Step 3 BS $u$ requests BS $u^{\prime}$ (or $u^{\prime \prime}$ ) for cooperation if (24) (or (25)) is met.

(1) BS $u^{\prime}$ (or $u^{\prime \prime}$ ) accepts the request if it has not accepted the cooperation requirement from another BS, giving $D_{u^{\prime}, m}=k$ (or $D_{u^{\prime \prime}, m}=k$ ). Then, go to Step 4.

(2) Otherwise, BS $u^{\prime}$ (or $u^{\prime \prime}$ ) refuses the request of BS $u$, and proceeds to Step 2 .

Step 4 BS $u$ sends the data of user $k$ to BS $u^{\prime}$ (or $u^{\prime \prime}$ ), and the two BSs carry out the STBC-based transmission to user $k$.

\section{Centralized Decision Making Assisted COOPERATION INTERCI Mitigation}

In this section, we propose another InterCI mitigation scheme called the centralized decision making assisted cooperation (CDMC). It motivates to make the best InterCI mitigation decisions, in order to maximize the sum rate of the users on a subcarrier, and also to improve the frequency reuse of the subcarriers. In addition to the assumptions made for the DDMC algorithm, the BSs operated under the CDMC are also assumed to share the "three-valued InterCI information" of the cell-edge users.

The DDMC algorithm is unable to always yield the best decisions because of the lack of InterCI information, such as the example in (28)(b). Inspired by this observation, the CDMC algorithm motivates to make the better decisions based on the three-valued InterCI information shared among the BSs while to keep the complexity low. Let us return the example of (21), where subcarrier $m$ is assumed to be allocated to users $k, k^{\prime}$ and $k^{\prime \prime}$ in cells $u, u^{\prime}$ and $u^{\prime \prime}$, respectively. In the CDMC, the three values for the InterCI suffered by, e.g., user $k$ from BS $u^{\prime}$ are defined as

$$
v_{u^{\prime}, k}= \begin{cases}-1 & \text { if } I_{u^{\prime}, k}<I_{o} \\ 0 & \text { if } I_{o} \leq I_{u^{\prime}, k}<I_{c} \\ 1 & \text { if } I_{u^{\prime}, k} \geq I_{c}\end{cases}
$$

where $I_{o}$ and $I_{c}$ are two new thresholds introduced for classifying the InterCI into three regions, which are 1) ignorable InterCI, when $v_{u^{\prime}, k}=-1$; 2) moderate InterCI, if $v_{u^{\prime}, k}=0$; and 3 ) strong InterCI, when $v_{u^{\prime}, k}=1$. Let the discrete InterCI among the three users be expressed as

$$
\begin{aligned}
\boldsymbol{V}_{m} & =\left[\begin{array}{ccc}
\nu_{k} & v_{u, k^{\prime}} & v_{u, k^{\prime \prime}} \\
v_{u^{\prime}, k} & \nu_{k^{\prime}} & v_{u^{\prime}, k^{\prime \prime}} \\
v_{u^{\prime \prime}, k} & v_{u^{\prime \prime}, k^{\prime \prime}} & \nu_{k^{\prime \prime}}
\end{array}\right] \\
& =\left[\begin{array}{lll}
\boldsymbol{v}_{k, m} & \boldsymbol{v}_{k^{\prime}, m} & \boldsymbol{v}_{k^{\prime \prime}, m}
\end{array}\right] .
\end{aligned}
$$

Here, $V_{m}$ is referred to as the discrete InterCI matrix, or simply the InterCI matrix, of subcarrier $m$, and $\boldsymbol{v}_{k, m}=$ $\left[\begin{array}{lll}\nu_{k} & v_{u^{\prime}, k} & v_{u^{\prime \prime}, k}\end{array}\right]^{T}$ is the InterCI vector of user $j$ on subcarrier $m$. In (30), a non-diagonal element explains the strength of the InterCI between a BS and a user, which is given by (29). By contrast, a diagonal element indicates whether the corresponding user has its SIR below or above the SIR threshold $\eta_{t}$, defined as

$$
\nu_{i}=\left\{\begin{array}{ll}
1 & \text { if } \eta_{i}<\eta_{t}, \\
0 & \text { if } \eta_{i} \geq \eta_{t},
\end{array} \quad i=k, k^{\prime}, k^{\prime \prime} .\right.
$$

Based on the InterCI matrix $V_{m}$ given by (30), the CDMC algorithm makes the decisions for a user according to the following four cases. 
- Case 0 (No Actions): When $\nu_{k}=\nu_{k^{\prime}}=\nu_{k^{\prime \prime}}=0$, meaning that the SIRs from BSs $u, u^{\prime}$ and $u^{\prime \prime}$ to users $k, k^{\prime}$ and $k^{\prime \prime}$ are all above the SIR threshold $\eta_{t}$. In this case, all BSs transmit data respectively to their users on subcarrier $m$.

- Case 1 (Cooperation): At least one of the three users on subcarrier $m$ satisfies the conditions:

$$
\begin{array}{r}
\nu_{k}=1 \& v_{u^{\prime}, k}=1 \& v_{u^{\prime \prime}, k} \neq 1, k \in \mathcal{K}^{(u)}, \\
u \neq u^{\prime} \neq u^{\prime \prime}, \forall u \in\{0,1,2\} .
\end{array}
$$

- Case 2 (Possible Cooperation): Any of the three users on subcarrier $m$ does not satisfy the conditions in (32), but at least one of the users satisfies the conditions:

$$
\begin{array}{r}
\nu_{k}=1 \& v_{u^{\prime}, k}=1 \& v_{u^{\prime \prime}, k}=1, k \in \mathcal{K}^{(u)}, \\
u \neq u^{\prime} \neq u^{\prime \prime}, \forall u \in\{0,1,2\} .
\end{array}
$$

- Case 3 (No Cooperation): Any of the three users on subcarrier $m$ does not satisfy the conditions of (32) and (33), but at least one of the users satisfies the conditions:

$$
\begin{array}{r}
\nu_{k}=1 \& v_{u^{\prime}, k} \neq 1 \& v_{u^{\prime \prime}, k} \neq 1, k \in \mathcal{K}^{(u)}, \\
u \neq u^{\prime} \neq u^{\prime \prime}, \forall u \in\{0,1,2\} .
\end{array}
$$

Let us below discuss the operations in the Cases of 1-3 in detail.

When the InterCI matrix $V_{m}$ belongs to Case 1, the CDMC algorithm is operated as the flow chart shown in Fig. 2. In this case, cooperative transmission for a user with its SIR below the SIR threshold $\eta_{t}$ can always be set up. In order to find the best cooperation option to maximize the sum rate of subcarrier $m$, as shown in Fig. 2, the decisions are made using three iterations indexed by $s$. Furthermore, for the sake of evaluating the quality of the decision made in an iteration, we introduce a metric $\varepsilon_{m}^{(s)}$ for the $s$ th iteration of subcarrier $m$. It can be shown that, in Case 1, there are three possible strategies for InterCI mitigation.

Strategy 1: Two BSs cooperate to transmit to a user, while the other BS stops transmission. In this case, we have $\varepsilon_{m}^{(s)}=1$, and the IMD variables are in the form of $D_{u, m}=k, D_{u^{\prime}, m}=$ $k, D_{u^{\prime \prime}, m}=-1$.

Strategy 2: Two BSs cooperate to transmit to a user, while the other BS transmits to its own user with the SIR below the SIR threshold $\eta_{t}$. In this case, we have $\varepsilon_{m}^{(s)}=2$ associated with the IMD variables taking the values as $D_{u, m}=k, D_{u^{\prime}, m}=k$, $D_{u^{\prime \prime}, m}=k^{\prime \prime}$.

Strategy 3: Two BSs cooperate to transmit to a user, while the other BS transmits to its own user with the SIR above the SIR threshold $\eta_{t}$. In this case, we have $\varepsilon_{m}^{(s)}=3$ the IMD variables taking the values as $D_{u, m}=k, D_{u^{\prime}, m}=k$, $D_{u^{\prime \prime}, m}=k^{\prime \prime}$.

As mentioned previously, the CDMC algorithm motivates to maximize the sum rate of subcarrier $m$ and the overall frequency reuse factor of the system. Hence, the algorithm makes the final decision in favor of these. Clearly, Strategy 1 has a very high probability to generate a smaller sum rate than Strategies 2 and 3, since Strategy 1 yields only one information transmission flow on subcarrier $m$. By contrast, Strategy 3 is the most desirable one, which has a much higher probability

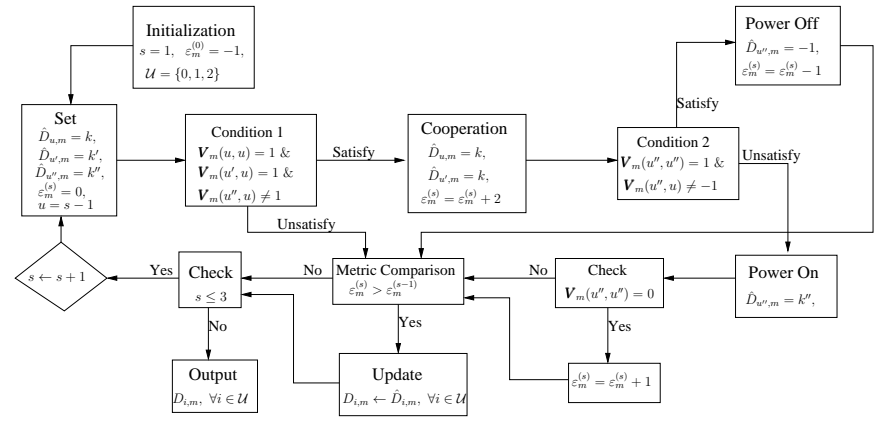

Fig. 2. Flow chart showing the operations of the CDMC algorithm in Case 1 , when assuming $u \neq u^{\prime} \neq u^{\prime \prime}$, and users $k, k^{\prime}, k^{\prime \prime}$ are in cells $u, u^{\prime}$ and $u^{\prime \prime}$, respectively.

than the other two strategies to obtain a higher sum rate. This is because Strategy 3 allows a cooperation between two BSs and another transmission from a BS to its user yielding a high SIR. Hence, the cooperation in Strategy 3 has the least cost.

Let us further use the example of (22) to explain, when $\eta_{t}=5 \mathrm{~dB}$ and $I_{c}=1, I_{o}=0.1$. Then, when the CDMC algorithm is used, the InterCI matrix is given by

$$
\boldsymbol{V}_{m}=\left[\begin{array}{ccc}
1 & -1 & 0 \\
1 & 1 & 0 \\
0 & 1 & 1
\end{array}\right]
$$

Explicitly, the operational situation is in Case 1 , as the conditions in (32) are met for both users $k$ and $k^{\prime}$.

According to the operations in Fig. 2, during the first $(s=1)$ iteration, the algorithm checks if a cooperation can be set up for user $k$. Since Condition 1 is met, a cooperation between BS $u$ and BS $u^{\prime}$ can be set up for user $k$. However, BS $u^{\prime \prime}$ has to turn off the transmission to user $k^{\prime \prime}$, as Condition 2 of $V_{m}(2,0)=0$ is satisfied. Consequently, from the first iteration, the decisions derived are $\hat{D}_{u, m}=k, \hat{D}_{u^{\prime}, m}=k$ and $\hat{D}_{u^{\prime \prime}, m}=-1$, which belong to Strategy 1 and have a metric of $\varepsilon_{m}^{(1)}=1$. During the second iteration, BS $u^{\prime}$ and BS $u^{\prime \prime}$ set up a cooperation for user $k^{\prime}$. Furthermore, user $k$ stays on because of $\boldsymbol{V}_{m}(0,1)=-1$. Therefore, from the second iteration, the decisions are $\hat{D}_{u, m}=k, \hat{D}_{u^{\prime}, m}=k^{\prime}$ and $\hat{D}_{u^{\prime \prime}, m}=k^{\prime}$, and the metric is $\varepsilon_{m}^{(1)}=2$. During the third iteration, the algorithm finds that it is unable to set up a cooperation for user $k^{\prime \prime}$. Therefore, the final InterCI mitigation decisions are given by the second iteration. It can be shown that, in this case, the sum rate achieved is $C_{\Sigma}=3.5213$, which is much higher than $C_{\Sigma}=2.208$ achieved by the DDMC.

Let us now address the operations of the CDMC algorithm operated under Case 2, the flow chart for which is shown in Fig. 3. There are two possible scenarios in Case 2. First, there is only one user, e.g., user $k$, having the SIR below $\eta_{t}$. In this case, as shown in Fig. 3, Condition 3 is satisfied and user $k$ suffers from two strong InterCI signals. Hence, due to the same reason for (26), the algorithm does not set up a cooperation for user $k$. Instead, it makes a decision about whether the transmission to user $k$ should be switched off or kept on. Specifically, the transmission to user $k$ is kept on, only when the transmission to it does not cause strong InterCI to the other two users, i.e., when Condition 4 is satisfied. Otherwise, 


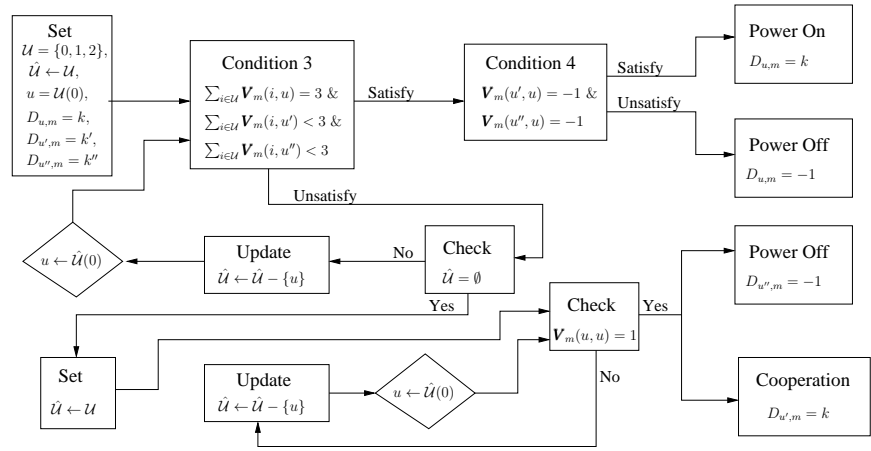

Fig. 3. Flow chart showing the operations of the CDMC algorithm in Case 2 , when assuming that $u \neq u^{\prime} \neq u^{\prime \prime}, u^{\prime}<u^{\prime \prime}$, and users $k, k^{\prime}, k^{\prime \prime}$ are in cells $u, u^{\prime}$ and $u^{\prime \prime}$, respectively.

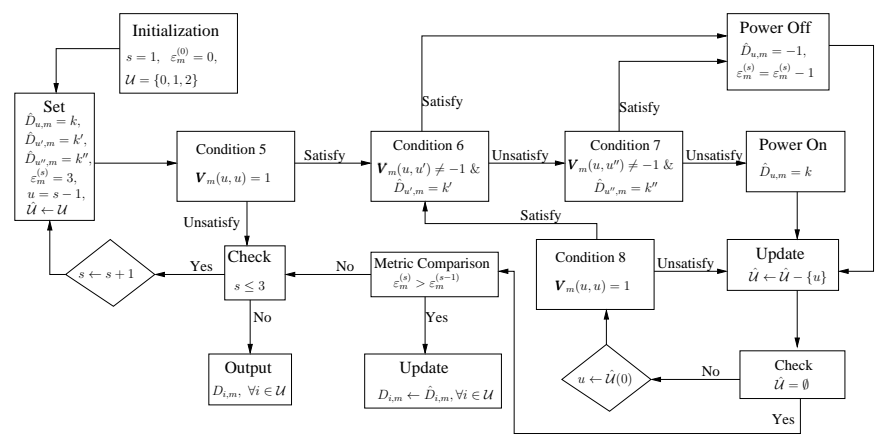

Fig. 4. Flow chart of Case 3 showing the operations of the CDMC algorithm, when assuming that $u \neq u^{\prime} \neq u^{\prime \prime}, u^{\prime}<u^{\prime \prime}$, and users $k, k^{\prime}, k^{\prime \prime}$ are in cells $u, u^{\prime}$ and $u^{\prime \prime}$, respectively.

the transmission to user $k$ is switched off. Second, there are more than one user having the SIR below $\eta_{t}$. In this scenario, a cooperation can be set up for a user, e.g., user $k$, with low SIR, while the transmission to the other user is switched off in order not to interfere the cooperation. Consequently, in Case 2 , there are two possible InterCI mitigation strategies, one is Strategy 1, which has been described under Case 1. The other one is Strategy 4 corresponding to the first scenario described above, which is stated as

Strategy 4: Switching off the transmission to one user, while keeping the transmission to the other two users, corresponding to the IMD variables in the form of $D_{u, m}=-1$, $D_{u^{\prime}, m}=k^{\prime}, D_{u^{\prime \prime}, m}=k^{\prime \prime}$.

Finally, let us consider the CDMC algorithm operated under Case 3 with the aid of Fig. 4. In this case, no cooperation for the users with poor SIR can be established, and the algorithm only needs to decide whether some transmissions should be switched off, in order to remove the strong InterCI imposing on the other users. As shown in Fig. 4, the final InterCI mitigation decisions can be made after three iterations to consider all the possible options. Similar to Case 1, here a metric $\varepsilon_{m}^{(s)}$ is introduced so as to evaluate the qualities of the decisions made during an iteration. As seen in Fig. 4, there are three optional decisions. The most desirable one is to keep all the three transmissions on subcarrier $m$, which gives a metric of $\varepsilon_{m}^{(s)}=3$. The next desirable decision is Strategy 4, which gives a metric of $\varepsilon_{m}^{(s)}=2$. The least desirable decision is given by Strategy 5, described as:

Strategy 5: Switching off two transmissions to two users, while remaining the other one on. Correspondingly, we have $\varepsilon_{m}^{(s)}=1$, and the IMD variables with the values of $D_{u, m}=$ $-1, D_{u^{\prime}, m}=-1, D_{u^{\prime \prime}, m}=k^{\prime \prime}$.

In summary, the principles of CDMC algorithm considering Cases 0-3 can now be described as follows.

\section{Algorithm 3: (CDMC Algorithm)}

\section{Initialization:}

(1) All users in the three cells estimate their SIRs: $\eta_{k, m}^{(u)}=\frac{\left|h_{k, m}^{(u)}\right|^{2}}{I_{u^{\prime}, k}+I_{u^{\prime \prime}, k}}$, if $k \in \mathcal{F}_{m}^{(u)} \& k^{\prime} \in \mathcal{F}_{m}^{\left(u^{\prime}\right)} \& k^{\prime \prime} \in$ $\mathcal{F}_{m}^{\left(u^{\prime \prime}\right)}$ and $m \in \mathcal{M} ; \forall k \in \mathcal{K}^{(u)}$ and $\forall u \in\{0,1,2\}$.

(2) Set $\mathcal{K}_{m}=\left\{k \mid k \in \mathcal{F}_{m}^{(u)}, \forall u \in\{0,1,2\}\right\}$, $\hat{\mathcal{K}}_{m}=\left\{k \mid \eta_{k}<\eta_{t}, k \in \mathcal{K}_{m}\right\}, \forall m \in \mathcal{M}$.

For subcarrier $m \in \mathcal{M}$ :

\section{If $\hat{\mathcal{K}}_{m} \neq \emptyset$, execute:}

Step 1 All discrete InterCI of the users in $\hat{\mathcal{K}}_{m}$ are sent to the head BS.

Step 2 Head BS asks for the discrete InterCI of all the users in $\mathcal{K}_{m}-\hat{\mathcal{K}}_{m}$. (Note that, after Steps 1 and 2, the head $B S$ has the knowledge of $\boldsymbol{V}_{m}$.)

Step 3 Based on $V_{m}$, the head BS makes the InterCI mitigation decisions based on the strategies in Cases 1 , 2 and 3, as described in Figs. 2 - 4.

Step 4 The head BS informs the other BSs the InterCI decisions by sending them the decisions of $\mathcal{D}_{m}$.

Note that, instead of letting a head BS make the decisions, we may let all the BSs make the decisions. In this way, there is no need for a BS to inform the other BSs its decisions, but all the BSs have to share the InterCI information for making decisions. Specifically, in this approach, when a BS knows that one of its users has the SIR below the threshold $\eta_{t}$, it then broadcasts the discrete InterCI vector of the user, such as the vector $\boldsymbol{v}_{k, m}$ in (30), to the other two BSs. Once receiving the InterCI vector, the other two BSs also broadcast the InterCI information of their users sharing the same subcarrier, regardless of the SIR values of their users. In this way, all the three BSs have the full knowledge of the discrete InterCI matrix of a subcarrier. Hence, they can make the same decisions in the principles of the CDMC under cases 1,2 or 3 .

So far, we have considered the principles of four types of InterCI mitigation algorithms, namely the FIIDM, OOP, DDMC and CDMC algorithms. In the context of a three-cell downlink OFDMA system, the InterCI mitigation is operated independently for the cell-edge user groups of each having three co-subcarrier users. We should note that these InterCI mitigation algorithms can all be modified for deployment in practical multicell systems, which may have a big number of cells and each cell may host an arbitrary number of users. First, owing to the structure of practical cellular systems, one user can usually simultaneously receive strong InterCI from at most two neighboring cells, which happens when a user is located the boarders of three cells. Therefore, even in practical multicell systems, one cell-edge user group contains only three co-subcarrier users. Furthermore, if the three co-subcarrier users in one group are not related to the other cell-edge user 
groups, then all the algorithms considered in our paper can be directly applied for InterCI mitigation. However, there is a possibility that one user is simultaneously a member of two or more cell-edge user groups. In this case, the InterCI algorithms can be modified to simply switch off the transmission to a user belonging to two or more cell-edge user groups. In fact, our proposed DDMC and CDMC algorithms can be readily modified to implement this operation. This can be achieved by switching off the transmission to one user on a subcarrier, whenever the user's serving BS receives two or more requests from other BSs for cooperation. Second, concerning the case that different cells may have different number of users, this only affects the subcarrier-allocation, but not the InterCI mitigation, as the InterCI mitigation only considers cell-edge users. However, when the number of subcarriers is higher than the number of users in a cell, one benefit is that a cell-edge user has an extra option to choose another subcarrier experiencing less InterCI. Nevertheless, this paper focuses on the InterCI mitigation, we hence avoid considering these trivial cases.

\section{PERFORMANCE RESUlts}

In this section, we provide a range of simulation results, in order to demonstrate and compare the achievable spectralefficiency performance of the multicell downlink OFDMA systems employing the BWSA subcarrier-allocation algorithm and the various InterCI mitigation algorithms. We assume that all subcarriers experience independent flat Rayleigh fading. The pathloss exponent in (4) is assumed to be $\mu=4.0$, and the standard deviation of the shadowing effect is $\Upsilon=8 \mathrm{~dB}$. Furthermore, for the sake of explicit comparison, we address the performance by focusing on the cell-edge users in the system. In the following figures, the average spectral-efficiency of cell-edge users per cell is given by

$$
C=\frac{1}{3} \sum_{u \in\{0,1,2\}} \sum_{k \in \tilde{\mathcal{K}}^{(u)}} \log _{2}\left(1+\gamma_{k}\right), \quad \text { (bits/s/Hz/cell). }
$$

Correspondingly, the average spectral-efficiency per cell-edge user is

$$
C=\frac{1}{|\tilde{\mathcal{K}}|} \sum_{u \in\{0,1,2\}} \sum_{k \in \tilde{\mathcal{K}}^{(u)}} \log _{2}\left(1+\gamma_{k}\right), \text { (bits/s/Hz/user) }
$$

where $\tilde{\mathcal{K}}=\tilde{\mathcal{K}}^{(0)} \cup \tilde{\mathcal{K}}^{(1)} \cup \tilde{\mathcal{K}}^{(2)}$, and $\tilde{\mathcal{K}}^{(u)}, u \in\{0,1,2\}$, is defined in (15). In (36) and (37), $\gamma_{k}$ is the SINR of user $k$, which is given by (6) or (7).

Fig. 5 compares the spectral-efficiency performance of the different InterCI mitigation algorithms employed by the threecell downlink OFDMA systems. From the results we can obtain the following observations. First, for all the considered SIR thresholds, both the proposed DDMC and CDMC algorithms yield higher spectral-efficiency than the OOP algorithm, and also higher than the case without InterCI mitigation, labeled as "non InterCI mitigation" in the figure. As shown in the figure, the DDMC and CDMC algorithms become more advantageous over the OOP algorithm as the threshold $\eta_{t}$ reduces. This is because, the DDMC and CDMC algorithms

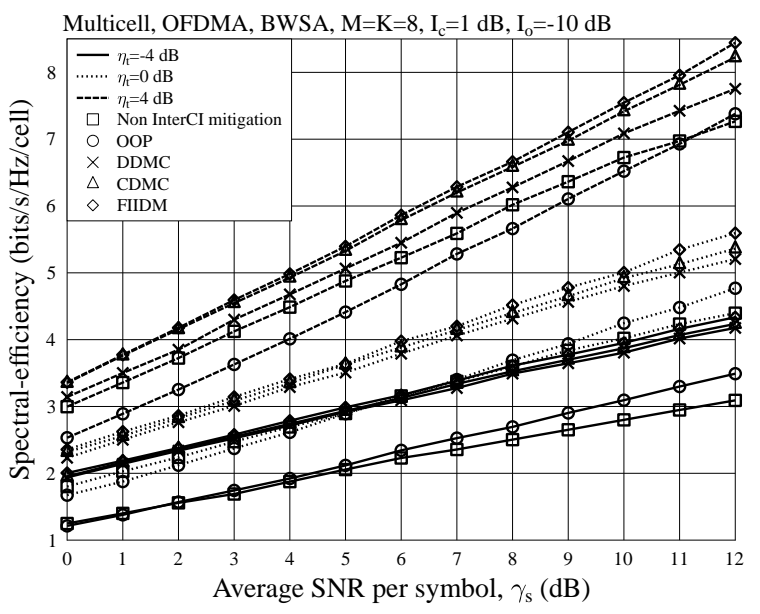

Fig. 5. Spectral-efficiency of cell-edge users in the multicell downlink OFDMA systems employing the BWSA subcarrier-allocation algorithm and various InterCI mitigation algorithms.

motivate to establish cooperative transmissions for the celledge users, instead of simply switching off. As $\eta_{t}$ reduces, the number of users requiring cooperation or switching off becomes less, meaning that the "edge-users" are closer to the cell's physical edge. In this case, setting up cooperation for the cell-edge users will be more beneficial than simply switching them off. Second, we can observe that the CDMC algorithm always outperforms the DDMC algorithm, and the gain becomes bigger as the SIR threshold $\eta_{t}$ increases. This is because, in the CDMC algorithm, the BSs find the joint InterCI mitigation decisions, while, in the DDMC algorithm, each BS makes distributed InterCI mitigation decisions only for its own users. Furthermore, the CDMC algorithm attains more SNR gain than the DDMC algorithm, when the number of cell-edge users increases, as a result of increase of the SIR threshold $\eta_{t}$. Third, Fig. 5 shows that the OOP algorithm may become useless in InterCI mitigation, when the SIR threshold is high, such as $\eta_{t}=4 \mathrm{~dB}$. In this case, there will be many users turned off. Fourth, as shown in Fig. 5, the OOP algorithm becomes more effective, when the average SNR gets larger. Therefore, when the system is too noisy or when the switching off threshold is too high, too many users may be switched off, which is not beneficial for the systems to use the OOP algorithm. Explicitly, the proposed DDMC and CDMC algorithms are capable of avoiding these drawbacks of the OOP algorithm, by setting up cooperation for celledge users, instead of simply turning off them. Lastly, we can observe that the spectral-efficiency performance attained by the CDMC algorithm is very close to that obtained by the FIIDM scheme, which uses the continuous InterCI information for decision making, while the CDMC algorithm only relies on the three-valued discrete InterCI information for decision making. As seen in the figure, the CDMC algorithm attains nearly the same spectral-efficiency as the FIIDM scheme when the average SNR is relatively low.

In Fig. 6, we investigate the average spectral-efficiency per active cell-edge user. First, we can observe that any of 


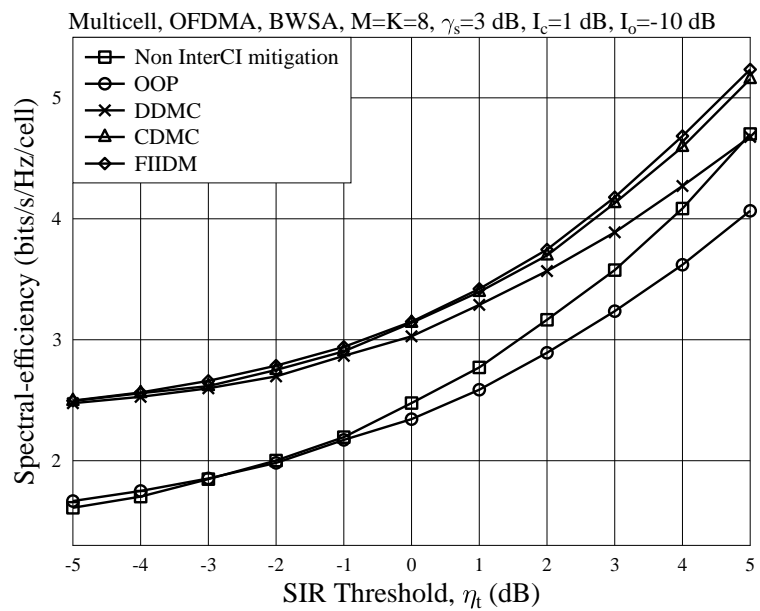

(a) $\gamma_{s}=3 \mathrm{~dB}$

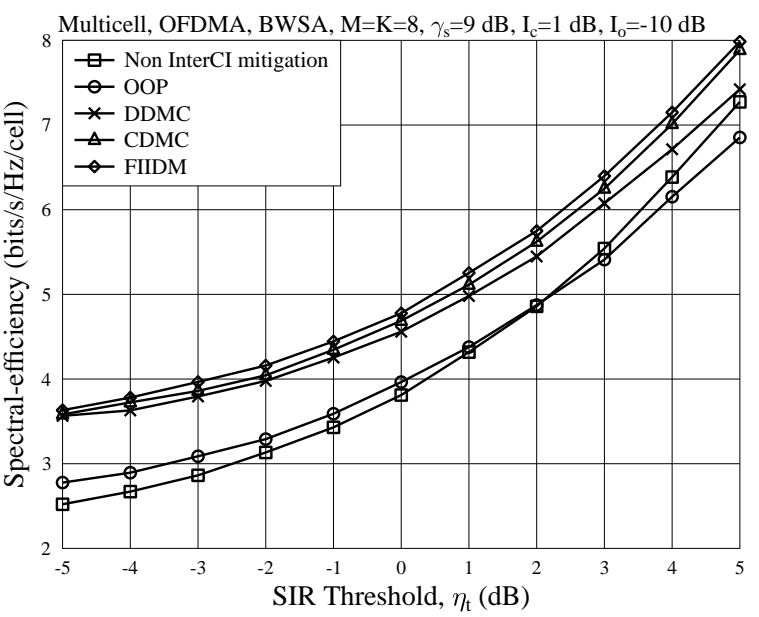

(b) $\gamma_{s}=9 \mathrm{~dB}$

Fig. 7. Comparison of spectral-efficiency performance of cell-edge users in the multicell downlink OFDMA systems employing various InterCI mitigation algorithms, when different SIR thresholds are applied.

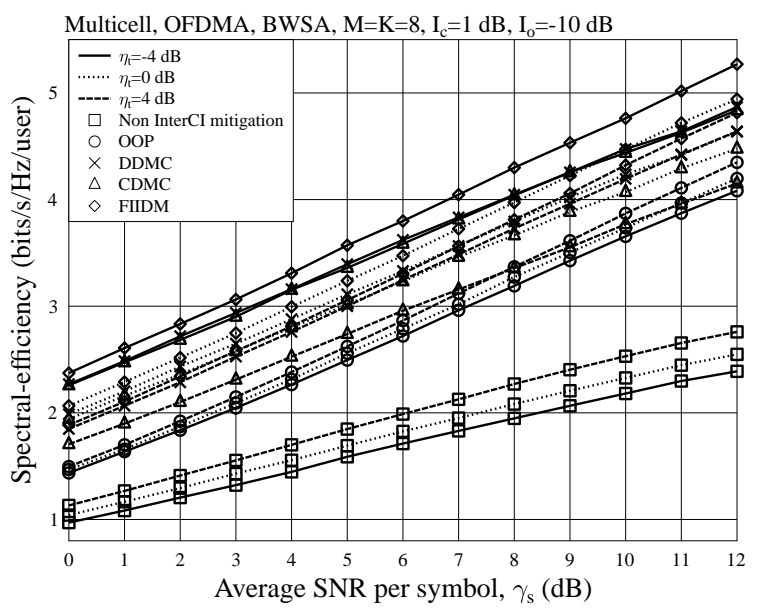

Fig. 6. Spectral-efficiency per active cell-edge user in the multicell downlink OFDMA systems employing the BWSA subcarrier-allocation algorithm and various InterCI mitigation algorithms.

the three InterCI mitigation schemes significantly outperforms the case of "non InterCI mitigation". Second, the CDMC algorithm achieves lower spectral-efficiency than the DDMC algorithm for all the SIR thresholds considered. The CDMC algorithm aims to maximize both the system's sum rate and the frequency reuse factor, while the DDMC algorithm is only sum rate motivated. Specifically, the DDMC algorithm simply switches off the transmission to the user when a cooperation is unavailable. By contrast, the CDMC algorithm still allows the transmission to the user, provided that this transmission does not cause strong InterCI to the other users. Consequently, given the same SIR threshold, the number of active cell-edge users resulted from the CDMC algorithm is higher than that resulted from the DDMC algorithm. This makes the average spectral-efficiency per active edge user attained by the CDMC algorithm smaller than that obtained by the DDMC algorithm. Finally, the FIIDM scheme yields the highest spectral-efficiency as seen in Fig. 6.

Fig. 7 compares the spectral-efficiency performance of the cell-edge users, when the SIR threshold varies in the range of $-5 \mathrm{~dB} \leq \eta_{t} \leq 5 \mathrm{~dB}$. From the figures we observe that the proposed DDMC and CDMC algorithms outperform the other two algorithms considered. As seen in the figures, the spectralefficiency performance of the proposed DDMC and CDMC algorithms as well as the OOP algorithm are all dependent on the SIR threshold applied. By comparing Fig. 7 (a) with Fig. 7 (b), we can see that the intersection between the curves of the OOP algorithm and the non InterCI mitigation case shifts from $\eta_{t}=-2 \mathrm{~dB}$ to $\eta_{t}=2 \mathrm{~dB}$, when the average SNR per symbol is increased from $\gamma_{s}=3 \mathrm{~dB}$ to $\gamma_{s}=9 \mathrm{~dB}$. Note that, as seen in Fig. 7, the spectral-efficiency in the case of "Non InterCI mitigation" also increases, as $\eta_{t}$ increases. This is because more users are considered as the cell-edge users, as $\eta_{t}$ increases, which makes the spectral-efficiency evaluated by (36) increases. Note furthermore that, at a given SNR, when $\eta_{t}$ increases, more users will be included as the cell-edge users, among which more users could be turned off, when the OOP algorithm is applied. This makes the spectral-efficiency of a cell achieved by the OOP algorithm become lower than that obtained by doing nothing. Furthermore, Fig. 7 once again shows that the proposed CDMC is capable of achieving the spectral-efficiency close to that of the FIIDM scheme.

In Fig. 8, we show the effect of the InterCI cooperation threshold $I_{c}$ and the off-power threshold $I_{o}$ on the spectralefficiency per cell, when the multicell downlink OFDMA systems employs the DDMC or CDMC algorithms. Explicitly, in Fig. 8 (a), for both the proposed algorithms, there are desirable $I_{c}$ values, which result in the highest spectral-efficiency. In general, when the threshold $I_{c}$ becomes smaller, the proposed algorithms try to establish cooperation for more users. By contrast, when $I_{c}$ becomes larger, they allow cooperation for fewer users. Note that, when $\eta_{t}=-4 \mathrm{~dB}$, Fig. 8 (a) shows that the highest spectral-efficiency per cell achieved by the DDMC 


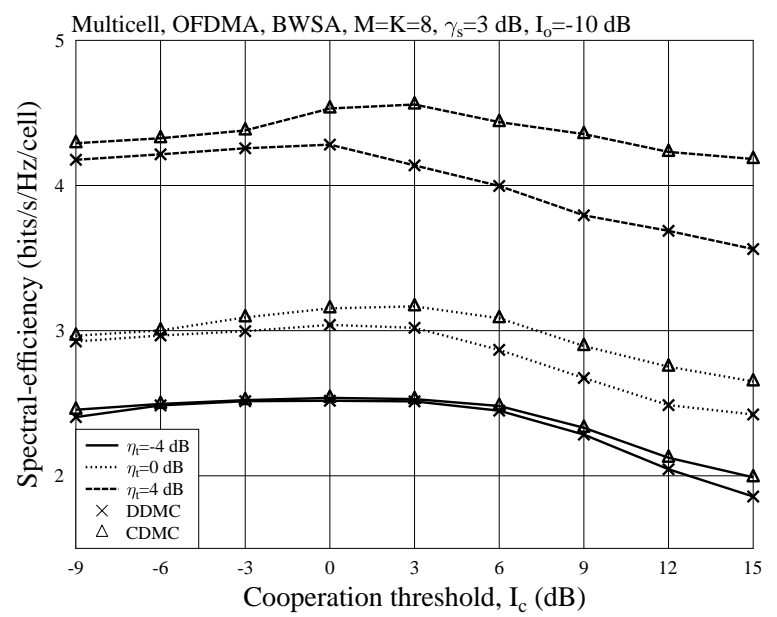

(a) Effect of $I_{c}$

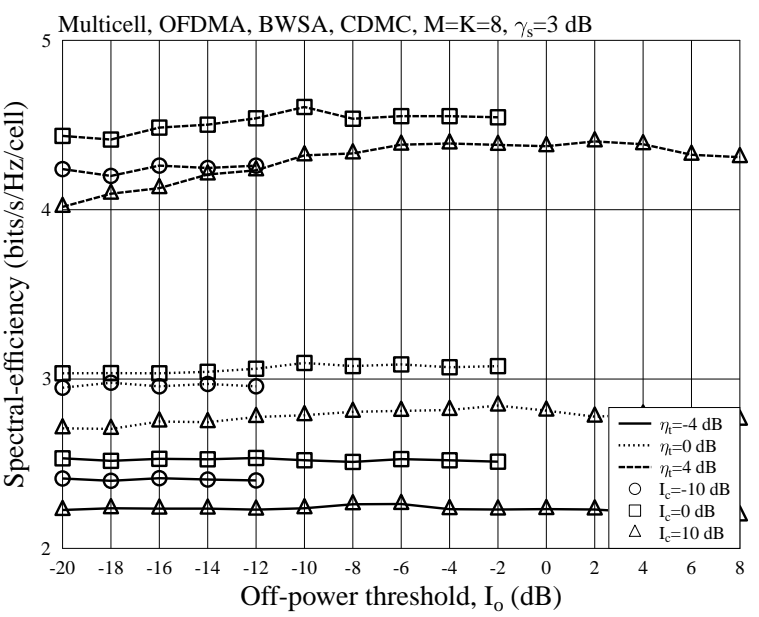

(b) Effect of $I_{o}$

Fig. 8. Comparison of spectral-efficiency performance of cell-edge users in the multicell downlink OFDMA systems employing various InterCI mitigation algorithms with different InterCI cooperation thresholds $I_{c}$ and off-power thresholds $I_{o}$.

and $\mathrm{CDMC}$ algorithms requires that $-6 \mathrm{~dB} \leq I_{c} \leq 6 \mathrm{~dB}$. However, the best $I_{c}$ range for the two algorithms is reduced to $-3 \mathrm{~dB} \leq I_{c} \leq 3 \mathrm{~dB}$ when $\eta_{t}=0 \mathrm{~dB}$, and to $-1 \mathrm{~dB} \leq$ $I_{c} \leq 1 \mathrm{~dB}$ when $\eta_{t}=4 \mathrm{~dB}$. This observation implies that the spectral-efficiency achieved by the two proposed algorithms becomes more sensitive to the cooperation threshold $I_{c}$, as the SIR threshold increases. In Fig. 8 (b), the results show that, at a low SIR threshold, such as $\eta_{t}=-4 \mathrm{~dB}$, the spectralefficiency per cell slightly varies, when different values of $I_{o}$ are employed. However, the CDMC algorithm yields a more explicit fluctuating spectral-efficiency per cell with respect to $I_{o}$, as the SIR threshold $\eta_{t}$ gets higher. Overall, we see that the spectral-efficiency achieved by the CDMC algorithm is not very sensitive to the InterCI off-power threshold $I_{o}$.

From Figs. 5-7, we may conclude that the SIR thresholds $\eta_{t}$ for both the DDMC and CDMC algorithms should be chosen according to the design objectives, so as to yield a good tradeoff between performance and complexity. From Fig. 8, we are implied that the threshold $I_{c}$ can be set to an appropriate value, so that a 'good' fraction of users experiencing strong InterCI are identified for BS cooperation, in order to improve the spectral-efficiency. Once the SIR threshold $\eta_{t}$ and the cooperation threshold $I_{c}$ are set, an off-power threshold $I_{o}$ can then be chosen within a relative large range of $I_{O}<I_{c}$ by the CDMC algorithm, as seen in Fig. 8(b).

In Figs. 9 and 10, we investigate the frequency reuse factor of the downlink OFDMA systems. Explicitly, the frequency reuse factor obtained by the CDMC algorithm is significantly higher than those given by the other algorithms. We also observe that the frequency reuse factor obtained by the CDMC algorithm increases sharply, as $\eta_{t}$ increases. By contrast, the frequency reuse factor achieved by the other two algorithms decreases, as $\eta_{t}$ increases. The above observations imply that, with the CDMC algorithm, the multicell downlink OFDMA system can simultaneously provide services for more users, even though some of them might have relatively low rates. By contrast, when the DDMC or OOP algorithm is employed,

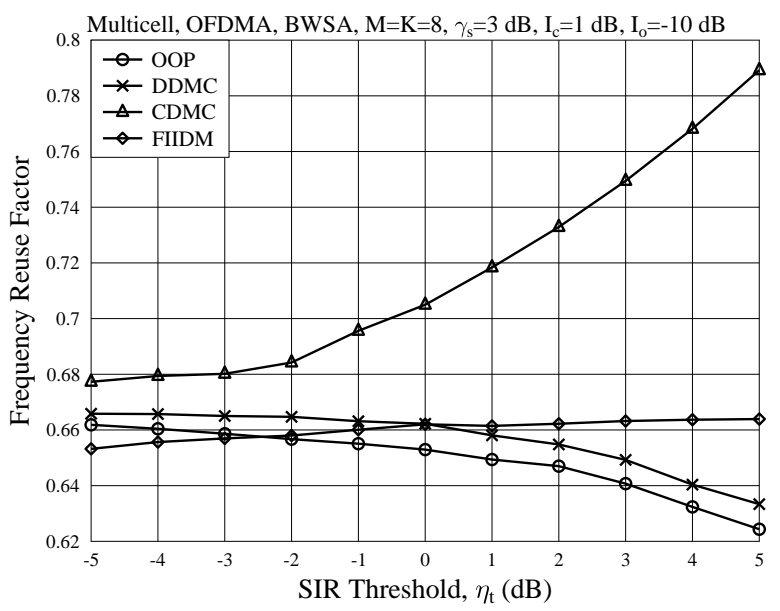

Fig. 9. Frequency reuse factor of cell-edge users in the multicell downlink OFDMA systems employing various InterCI mitigation algorithms with respect to different SIR thresholds $\eta_{t}$.

the number of users switched off increases as $\eta_{t}$ increases, which results in the drop of the frequency reuse factor. Figs. 9 shows that the frequency reuse factor achieved by the DDMC algorithm is slightly higher than that obtained by the OOP algorithm, owing to the cooperation introduced in the DDMC algorithm. Additionally, as seen in Fig. 9, the FIIDM algorithm yields a lower frequency factor than the DDMC and OOP algorithms in the low $\eta_{t}$ regimes. This means that, in order to maximize the spectral-efficiency, the FIIDM algorithm has to turn off the transmissions with poor SIR.

Fig. 10 shows that the frequency reuse factor obtained by the CDMC algorithm increases towards one, as the InterCI cooperation threshold $I_{c}$ increases. This is because, when the cooperation threshold $I_{c}$ is set higher, it will be more difficult for the CDMC algorithm to establish cooperation for cell-edge users. Therefore, more cell-edge users will be 


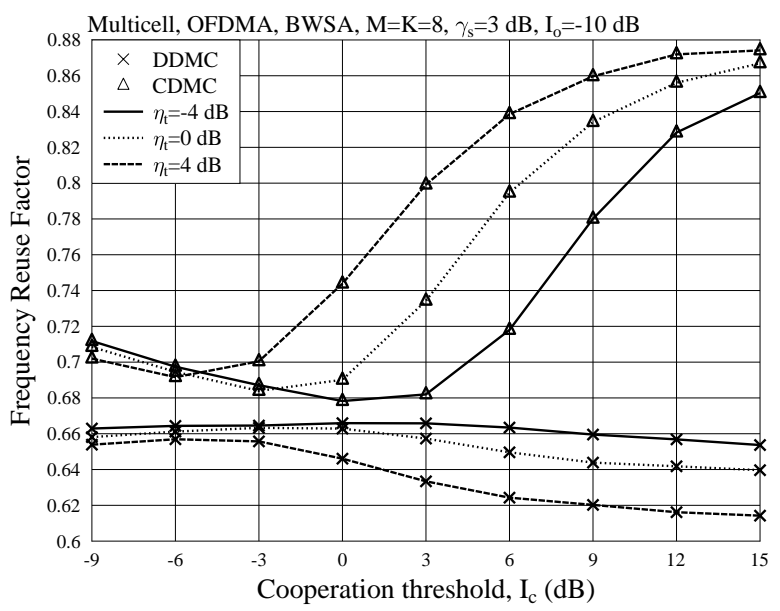

Fig. 10. Frequency reuse factor of cell-edge users in the multicell downlink OFDMA systems employing various InterCI mitigation algorithms with respect to different InterCI cooperation thresholds $I_{C}$.

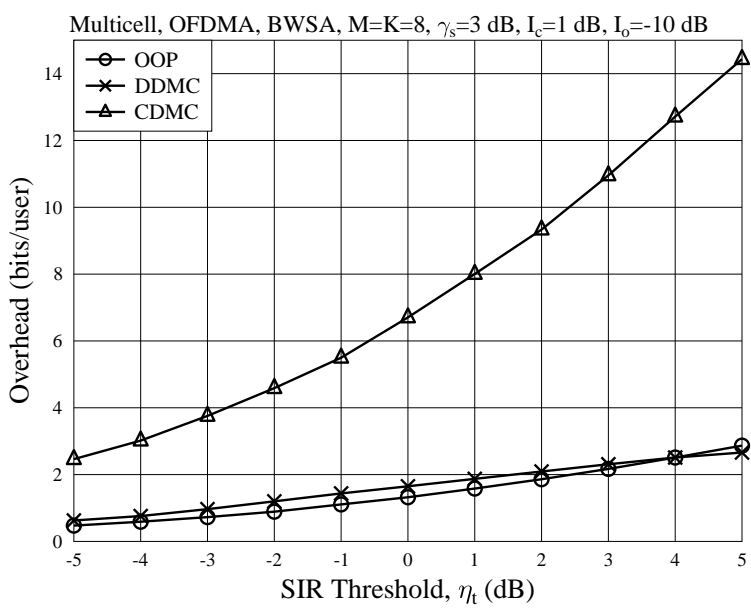

Fig. 11. Overhead required by the various InterCI mitigation algorithms.

kept on. Furthermore, as the figure shows, when $I_{c} \leq 0 \mathrm{~dB}$, the frequency reuse factor achieved by the CDMC algorithm slightly decreases, as the SIR threshold increases. For the DDMC algorithm, as shown in Fig. 10, the frequency reuse factor slightly decreases, as the threshold $I_{c}$ increases. This is the result that the DDMC algorithm turns off more users, when the threshold $I_{c}$ becomes higher.

Explicitly, the operations of the OOP, DDMC and CDMC algorithms require different overhead. Hence, in Fig. 11, we compare the overhead required by the various InterCI mitigation algorithms. Here, the overhead is measured by the number of bits per user, which is obtained from the total overhead (bits) of a cell divided by the number of users in the cell. The overhead considered includes the control information transmitted between users and their BSs and those among BSs, plus the data symbols shared among the BSs for cooperation. For all the three InterCI mitigation algorithms, we assume that one bit is required to transmit a request for cooperation or off-power. Furthermore, in Fig. 11, we assume that, under the CDMC algorithm, the decisions are made by the head BS, as described in Algorithm 3. The discrete InterCI vector of a subcarrier, such as $\boldsymbol{v}_{k, m}$ in (30), has 18 different states. Hence, a BS needs 4 bits to convey the discrete InterCI vector of a subcarrier. Therefore, in total 8 bits of overhead are required for the two BSs to inform the head BS their InterCI information of a subcarrier. In addition, another 3 bits are required for the head $\mathrm{BS}$ to broadcast the InterCI mitigation decisions of a subcarrier to the other two BSs, since the decisions have 9 states in total. As the number of cell-edge users increases, when the SIR threshold gets higher, Fig. 11 correspondingly shows that the required overhead for all the three algorithms increases, as the SIR threshold becomes higher. Furthermore, the CDMC algorithm requires higher overhead than the other two algorithms. However, the DDMC algorithm requires very low overhead, which is similar to that required by the OOP algorithm.

\section{CONCLUSIONS}

In this paper, we have proposed the DDMC and CDMC algorithms for mitigating the InterCI among the cell-edge users sharing the same subcarrier. While both the DDMC and CDMC InterCI mitigation algorithms motivate to maximize the spectral-efficiency, the CDMC algorithm also aims to maximize the frequency reuse factor. In this paper, we have compared from different perspective the achievable performance of the downlink OFDMA systems employing the various InterCI mitigation schemes. Our studies and performance results show that both the DDMC and CDMC algorithms are capable of achieving higher spectral-efficiency than the OOP algorithm, and, certainly, than the case without employing any InterCI mitigation. Although only the three-valued discrete InterCI information is shared among the BSs, the CDMC algorithm is capable of attaining nearly the same performance as the optimal FIIDM scheme that uses the continuous InterCI information for decision making. Additionally, the CDMC algorithm is demonstrated to have the highest frequency reuse factor in addition to its spectral-efficiency advantage, whereas, the DDMC algorithm requires a small amount of overhead, which is similar to that of the OOP algorithm.

\section{REFERENCES}

[1] J. Jang and K. B. Lee, "Transmit power adaptation for multiuser OFDM systems," IEEE Journal on Selected Areas in Communications, vol. 21, no. 2, pp. $171-178$, Feb. 2003.

[2] K. Kim and J. Kim, "A 2-D subcarrier allocation scheme for capacity enhancement in a clustered OFDM system," IEICE Transactions on Communications, vol. E90-B, pp. 1880 - 1883, July 2007.

[3] K. A. D. Teo, Y. Otani, and S. Ohno, "Adaptive subcarrier allocation for multi-user OFDM system," IEICE Transactions on Communications, vol. E89-A, pp. 3131 - 3137, July 2006.

[4] N. Y. Ermolova and B. Makarevitch, "Performance of practical subcarrier allocation schemes for OFDM," Proceedings of IEEE International Symposium on Personal, Indoor and Mobile Radio Communications (PIMRC), pp. 1 - 4, Sept. 2007.

[5] O. Nwamadi, X. Zhu, and A. Nandi, "Dynamic subcarrier allocation for single carrier - FDMA systems," in Proceedings of EUSIPCO-2008, Aug. 2008.

[6] T. Liu, C. Yang, and L.-L. Yang, "A low-complexity subcarrier-power allocation scheme for frequency-division multiple-access systems," IEEE Transactions on Wireless Communications, vol. 9, no. 5, pp. 1564 1570, May 2010. 
[7] T. Wang and L. Vandendorpe, "Iterative resource allocation for maximizing weighted sum min-rate in downlink cellular OFDMA systems,' IEEE Transactions on Signal Processing, vol. 59, no. 1, pp. 223-234, Jan. 2011.

[8] M. Abaii, Y. Liu, and R. Tafazolli, "An efficient resource allocation strategy for future wireless cellular systems," IEEE Transactions on Wireless Communications, vol. 7, no. 8, pp. 2940 - 2949, Aug. 2008.

[9] S. M. H. Andargoli and K. Mohamed-Pour, "Weighted sum throughput maximisation for downlink multicell orthogonal frequency-division multiple access systems by intercell interference limitation," IET Communications, vol. 6, no. 6, pp. 628 - 637, April 2012.

[10] D. W. K. Ng, E. S. Lo, and R. Schober, "Energy-efficient resource allocation in multi-cell OFDMA systems with limited backhaul capacity," IEEE Transactions on Wireless Communications, vol. 11, no. 10, pp. 3618 - 3631, Oct. 2012

[11] L. Venturino, A. Zappone, C. Risi, and S. Buzzi, "Energy-efficient scheduling and power allocation in downlink OFDMA networks with base station coordination," IEEE Transactions on Wireless Communications, vol. 14, no. 1, pp. 1-14, Jan. 2015.

[12] E. Baktash, M. Rasti, and E. Hossain, "Resource allocation for dynamic intra-cell subcarrier reuse in cooperative OFDMA wireless networks," IEEE Transactions on Mobile Computing, vol. 14, no. 7, pp. 1475-1489, July 2015.

[13] M. Fathi and E. Karipidis, "Distributed resource optimization in multicell OFDMA networks," Proceedings of IEEE Wireless Communications and Networking Confernce (WCNC-2012), pp. 1316 - 1320, April 2012

[14] K. W. Choi, E. Hossain, and D. I. Kim, "Downlink subchannel and power allocation in multi-cell OFDMA cognitive radio networks," IEEE Transactions on Wireless Communications, vol. 10, no. 7, pp. 22592271, July 2011.

[15] B. Ozbek, D. L. Ruyet, and M. Pischella, "Adaptive reduced feedback links for distributed power allocation in multicell MISO-OFDMA networks," IEEE Wireless Communications Letters, vol. 3, no. 2, pp. 141144, April 2014.

[16] D. W. K. Ng and R. Schober, "Resource allocation and scheduling in multi-cell OFDMA systems with decode-and-forward relaying," IEEE Transactions on Wireless Communications, vol. 10, no. 7, pp. 2246 2258, July 2011.

[17] M. Moretti, A. Todini, A. Baiocchi, and G. Dainelli, "A layered architecture for fair resource allocation in multicellular multicarrier systems," IEEE Transactions on Vehicular Technology, vol. 60, no. 4, pp. 17881798, May 2011.

[18] Y. Yu, E. Dutkiewicz, X. Huang, and M. Mueck, "Downlink resource allocation for next generation wireless networks with inter-cell interference," IEEE Transactions on Wireless Communications, vol. 12, no. 4 pp. 1783-1793, April 2013.

[19] L. Venturino, N. Prasad, and X. Wang, "Coordinated scheduling and power allocation in downlink multicell OFDMA networks," IEEE Transactions on Vehicular Technology, vol. 58, no. 6, pp. 2835-2848, July 2009.

[20] H. Zhang, L. Venturino, N. Prasad, P. Li, S. Rangarajan, and X. Wang, "Weighted sum-rate maximization in multi-cell networks via coordinated scheduling and discrete power control," IEEE Journal on Selected Areas in Communications, vol. 29, no. 6, pp. 1214-1224, June 2011.

[21] S. H. Ali and V. C. M. Leung, "Dynamic frequency allocation in fractional frequency reused OFDMA networks," IEEE Transactions on Wireless Communications, vol. 8, no. 8, pp. 4286 - 4295, Aug. 2009.

[22] H. Galeana-Zapien and R. Ferrus, "Design and evaluation of a backhaulaware base station assignment algorithm for OFDMA-based cellular networks," IEEE Transactions on Wireless Communications, vol. 9, no. 10 , pp. 3226-3237, Oct. 2010.

[23] A. Chowdhery, W. Yu, and J. M. Cioffi, "Cooperative wireless multicell OFDMA network with backhaul capacity constraints," Proceedings of IEEE International Conference on Communications (ICC-2011), pp. 16, June 2011.

[24] Z. Hasan, G. Bansal, E. Hossain, and V. K. Bhargava, "Energy-efficient power allocation in OFDM-based cognitive radio systems: A risk-return model," IEEE Transactions on Wireless Communications, vol. 8, no. 12, pp. 6078-6088, Dec. 2009.

[25] X. Xiao, X. Tao, Y. Jia, and J. Lu, "An energy-efficient hybrid structure with resource allocation in OFDMA networks," Proceedings of IEEE Wireless Communications and Networking Conference (WCNC-2011), pp. 1466-1470, March 2011.

[26] J. Shi and L. liang Yang, "Bidirectional worst subchannel avoiding versus best subchannel seeking subcarrier-allocation in downlink OFDMA systems," IEEE Transactions on Vehicular Technology, Early Access, 2015 .
[27] C. Y. Ho and C.-Y. Huang, "Non-cooperative multi-cell resource allocation and modulation adaptation for maximizing energy efficiency in uplink OFDMA cellular networks," IEEE Wireless Communications Letters, vol. 1, no. 5, pp. 420-423, 2012.

[28] S.-Y. Kim, J.-A. Kwon, and J.-W. Lee, "Sum-rate maximization for multicell OFDMA systems," IEEE Transactions on Vehicular Technology, vol. 64, no. 9, pp. 4158-4169, 2015.

[29] B. Da and R. Zhang, "Exploiting interference alignment in multi-cell cooperative OFDMA resource allocation," in IEEE Global Telecommunications Conference (GLOBECOM 2011), 2011, pp. 1-5.

[30] A. J. Viterbi, A. M. Viterbi, and E. Zehavi, "Other-cell interference in cellular power-controlled CDMA," IEEE Transactions on Communications, vol. 42, no. 234, pp. 1501 - 1504, Feb. 1994.

[31] D. Gesbert, S. G. Kiani, A. Gjendemsjo, and G. E. Oien, "Adaptation, coordination, and distributed resource allocation in interference-limited wireless networks," Proceedings of the IEEE, vol. 95, no. 12, pp. 2393 2409, Dec. 2007.

[32] S. Alamouti, "A simple transmit diversity technique for wireless communications," IEEE Journal on Selected Areas in Communications, vol. 16 no. 8, pp. 1451 - 1458, Oct. 1998

[33] J. Shi and L.-L. Yang, "Novel subcarrier-allocation schemes for downlink MC DS-CDMA systems," IEEE Transactions on Wireless Communications, vol. 13, no. 10, pp. 5716-5728, Oct. 2014.

[34] A. Gjendemsjo, D. Gesbert, G. E. Oien, and S. G. Kiani, "Optimal power allocation and scheduling for two-cell capacity maximization," Proceedings of International Symposium on Modeling and Optimization in Mobile, Ad Hoc and Wireless Networks, pp. 1-6, April 2006.

[35] S. G. Kiani, G. E. Oien, and D. Gesbert, "Maximizing multicell capacity using distributed power allocation and scheduling," Proceedings of IEEE Wireless Communications and Networking Conference (WCNC-2007), pp. 1690-1694, March 2007. 